\title{
Multidrug Resistant Acinetobacter baumannii Biofilms: Evaluation of Phenotypic-Genotypic Association and Susceptibility to Cinnamic and Gallic Acids
}

\author{
Mahmoud M. Sherif', Walid F. Elkhatib ${ }^{2,3 *}$, Wafaa S. Khalaf ${ }^{4}$, Nooran S. Elleboudy ${ }^{2}$ and \\ Neveen A. Abdelaziz' \\ 1 Department of Microbiology and Immunology, Faculty of Pharmacy, Ahram Canadian University, 6th of October City, Egypt, \\ ${ }^{2}$ Department of Microbiology and Immunology, Faculty of Pharmacy, Ain Shams University, Cairo, Egypt, ${ }^{3}$ Department of \\ Microbiology and Immunology, Faculty of Pharmacy, Galala University, Al Galala, Egypt, ${ }^{4}$ Department of Microbiology and \\ Immunology, Faculty of Pharmacy (Girls), Al-Azhar University, Cairo, Egypt
}

\section{OPEN ACCESS}

Edited by:

Lene Karine Vestby,

Norwegian Veterinary Institute (NVI),

Norway

Reviewed by:

Sunil D. Saroj,

Symbiosis International University,

India

Sueli Fumie Yamada-Ogatta, State University of Londrina, Brazil

*Correspondence:

Walid F. Elkhatib

walid-elkhatib@pharma.asu.edu.eg; walid2005faisa/@yahoo.com orcid.org/0000-0001-5815-3200

Specialty section:

This article was submitted to Antimicrobials, Resistance and Chemotherapy,

a section of the journal

Frontiers in Microbiology

Received: 28 May 2021

Accepted: 17 August 2021

Published: 17 September 2021

Citation:

Sherif MM, Elkhatib WF, Khalaf WS, Elleboudy NS and

Abdelaziz NA (2021) Multidrug Resistant Acinetobacter baumannii Biofilms: Evaluation of Phenotypic-Genotypic Association and Susceptibility to Cinnamic and

Gallic Acids.

Front. Microbiol. 12:716627. doi: 10.3389/fmicb.2021.716627
Acinetobacter baumannii armed with multidrug resistance (MDR) and biofilm-forming ability is increasingly recognized as an alarming pathogen. A deeper comprehension of the correlation between these two armories is required in circumventing its infections. This study examined the biofilm-forming ability of the isolates by crystal violet staining and the antibiotic susceptibility by broth microdilution method. The genetic basis of the MDR and biofilm-forming phenotypes was screened by polymerase chain reaction. The antimicrobial activities of cinnamic and gallic acids against planktonic cells and biofilms of $A$. baumannii were investigated, and the findings were confirmed with scanning electron microscopy (SEM). Among 90 A. baumannii isolates, 69 (76.6\%) were MDR, and all were biofilm formers; they were classified into weak (12.2\%), moderate (53.3\%), and strong (34.5\%) biofilm formers. Our results underlined a significant association between MDR and enhanced biofilm formation. Genotypically, the presence of blavIM and blaOXA-23 genes along with biofilm-related genes (ompA, bap, and csuE) was statistically associated with the biofilm-forming abilities. Impressively, both gallic and cinnamic acids could significantly reduce the MDR $A$. baumannii biofilms with variable degrees dependent on the phenotype-genotype characteristics of the tested isolates. The current findings may possess future therapeutic impact through augmenting antimicrobial arsenal against life-threatening infections with MDR A. baumannii biofilms.

Keywords: Acinetobacter baumannii, biofilm, correlation, multidrug resistance, cinnamic acid, gallic acid

\section{INTRODUCTION}

Acinetobacter baumannii is receiving considerable attention as a troublesome pathogen owing to its extensive resistance to nearly all commonly used antimicrobials (Elkhatib et al., 2019), in addition to carbapenems, which are usually reserved to combat multidrug resistant (MDR) isolates (Xie et al., 2020). Carbapenem-resistant A. baumannii (CRAB) is ranked first priority by the World Health Organization (WHO) as a critical pathogen that urgently needs novel antimicrobial therapeutic strategies (WHO, 2017). Moreover, Centers for Disease Control and Prevention (CDC) has described CRAB as an "urgent threat" (CDC, 2019). During the coronavirus disease 2019 
(COVID-19) pandemic, A. baumannii has added to the toll by causing fatal ventilator-associated pneumonia (VAP) outbreaks in COVID-specific intensive care units (ICUs) (Gottesman et al., 2021). Carbapenem-hydrolyzing $\beta$-lactamase genes, chromosomal or plasmid mediated, are the most common mechanism of carbapenem resistance in $A$. baumannii comprising bla $a_{\mathrm{OXA}-23,}$ bla $a_{\mathrm{OXA}-24}, \quad b l a_{\mathrm{OXA}-51,}$ bla $a_{\mathrm{OXA}-58}$, bla $a_{\mathrm{NDM}}$, bla $a_{\mathrm{VIM}}$, and bla $a_{\mathrm{IMP}}$ (Lee et al., 2017). The high transmissibility of A. baumannii in hospital settings is greatly aided by its biofilm-forming capacity (Asaad et al., 2021). Biofilms offer protection to pathogens in the face of external stressors. Consequently, such infections respond inconsistently to antimicrobial treatments (Vestby et al., 2020). Genetic determinants related to biofilm include the biofilmassociated protein encoded by the bap gene, the outer membrane protein A (ompA), the pilus-like bundle structure mediated by the $c s u \mathrm{E}$ gene, and bla $a_{\mathrm{PER}-1}$ belonging to the $\beta$-lactamase family (Yang et al., 2019). Nonetheless, the interplay between biofilms and antimicrobial resistance of MDR A. baumannii isolates is rather disputable. Some researchers proved that A. baumannii expressed high levels of resistance to antibiotics despite producing weak biofilms (Qi et al., 2016), while others reported a positive correlation between antimicrobial resistance and biofilm-forming ability in MDR A. baumannii isolates (Bardbari et al., 2017).

In light of this combined problem, various approaches are implied to control spreading of biofilm-forming MDR A. baumannii strains (Farhadi et al., 2019). Lately, deployment of polyphenols is rising in popularity as a safe antibacterial and antibiofilm strategy (Zhang et al., 2019). Phenolic acids, such as gallic and cinnamic acid derivatives, cause irreversible changes in microbial membrane properties resulting in leakage of essential intracellular constituents (Kang et al., 2018). Concerning their antibiofilm activity, phenolic acids are being screened for "quorum quenching" abilities to disrupt quorum-sensing signals. Quorum sensing (QS) aids biofilm formation, rendering it a potential target for antibiofilm agents (Zhang et al., 2020).

There is a pressing need to comprehensively understand the nature of biofilms in A. baumannii, hence developing innovative and effective drugs to control such resistant infections. Accordingly, our study aimed to assess the relationship between biofilm formation and phenotypic-genotypic antibiotic resistance patterns in clinical isolates of A. baumannii. Besides, estimating the dissemination of biofilm-related genes with an attempt to pinpoint key ones serving as a predictor of strong biofilm formation phenotype. In addition, we investigated the antimicrobial and antibiofilm activities of cinnamic and gallic acids on MDR A. baumannii with a focus on underlying aspects influencing their effects.

\section{MATERIALS AND METHODS}

\section{Bacterial Isolates}

In this study, previously identified and stocked clinical A. baumannii isolates $(n=90)$, during the period March 2018-2019, were obtained from the Microbiology laboratory in El-Demerdash hospital, Faculty of Medicine, Ain Shams
University (Cairo, Egypt). Identification was verified by detecting

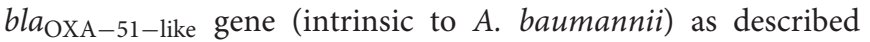
previously (El Far et al., 2019). A. baumannii ATCC 19606 was used as a positive control. All isolates were preserved in trypticase soy broth medium (TSB, Difco ${ }^{\circledR}$, Franklin Lakes, NJ, United States) with $15 \%$ glycerol, at $-80^{\circ} \mathrm{C}$ for subsequent uses (Abdel-Baky et al., 2017).

\section{Antibiogram of $A$. baumannii Isolates}

Five antibiotics were selected with different mechanisms of action: doxycycline (Sedico Co., Giza, Egypt) and amikacin (Eipico Co., Tenth of Ramadan City, Egypt) interrupting protein synthesis, imipenem (Merck \& Co., Kenilworth, NJ, United States) inhibiting cell wall synthesis, levofloxacin (Sedico Co., Giza, Egypt) inhibiting cell division, and colistin (Sedico Co., Giza, Egypt) disrupting the outer cell membrane. Microbroth dilution method was used to determine the minimum inhibitory concentrations (MICs) according to the Clinical and Laboratory Standards Institute (CLSI) guidelines (CLSI, 2018). Negative control wells contained only culture media to ensure sterility, while positive controls were inoculated with the organism, and Escherichia coli ATCC 25922 was used as a reference strain. Following a $24-\mathrm{h}$ incubation at $37^{\circ} \mathrm{C}, 30-\mu \mathrm{l}$ aliquots of $0.015 \%$ resazurin solution (Thermo Fisher Scientific, Waltham, MA, United States) were added to each well and further incubated for $2 \mathrm{~h}$. Change in resazurin color from blue to pink indicates bacterial growth (Elshikh et al., 2016). The susceptibility patterns of the isolates were interpreted according to the CLSI M07-A11 protocol (CLSI, 2019).

\section{Microtiter Plate Biofilm Formation Assay}

Biofilm formation was screened as described by Lee et al. (2008). In brief, overnight cultures in trypticase soya broth (Hi-Media, Mumbai, India) were used to prepare inoculum equivalent to $0.5 \mathrm{McFarland}$ standard adjusted spectrophotometrically at A600, then diluted 1:20 in trypticase soya broth. Aliquots $(200 \mu \mathrm{l})$ of each bacterial suspension were dispensed in three wells of a 96-well flat-bottomed microtiter plate (Corning, Corning, NY, United States) and incubated for $24 \mathrm{~h}$ at $37^{\circ} \mathrm{C}$. After incubation, the bacterial suspensions were carefully aspirated; then, wells were (i) washed with $200 \mu \mathrm{l}$ of phosphate-buffered saline (PBS) to remove planktonic cells, (ii) decanted and air dried for $15 \mathrm{~min}$, and (iii) stained with $200 \mu \mathrm{l}$ of $0.1 \% \mathrm{v} / \mathrm{v}$ crystal violet solution and left to stand for $15 \mathrm{~min}$. Then, each well was washed with PBS to remove excess stain. Finally, the stained wells were solubilized with $200 \mu \mathrm{l}$ of $33 \% \mathrm{v} / \mathrm{v}$ acetic acid and incubated at $37^{\circ} \mathrm{C}$ for $15 \mathrm{~min}$, and the optical density was measured at $630 \mathrm{~nm}$ using microtiter plate reader (ELx800, Biotek, Winooski, VT, United States). Uninoculated wells served as negative controls, and A. baumannii ATCC19606 was used as a positive control for the biofilm formation. Results were recorded as means of absorbance readings from triplicate wells. As described previously (Lee et al., 2008), the OD cutoff value $\left(O D_{c}\right)$ for biofilm formation was defined as three standard deviations above the mean absorbance of the inoculum free negative control $\left(O D_{\mathrm{c}}=O D_{\text {avg. of negative control }}+3\right.$ standard deviation (SD) of negative control). Accordingly, isolates were classified as non-biofilm formers when $O D \leq O D_{\mathrm{c}}$, weak biofilm 
formers when $O D_{\mathrm{c}}<O D \leq\left(2 \times O D_{\mathrm{c}}\right)$, moderate biofilm formers when $2 \times O D_{\mathrm{c}}<O D \leq\left(4 \times O D_{\mathrm{c}}\right)$, and strong biofilm formers when $O D>\left(4 \times O D_{\mathrm{c}}\right)$.

\section{PCR Amplification of $\beta$-Lactamase and Biofilm-Related Genes}

Genomic DNA was extracted using GeneJet ${ }^{\mathrm{TM}}$ genomic DNA extraction kit (Thermo Fisher Scientific, Waltham, MA, United States) according to the manufacturer's instructions and stored at $-20^{\circ} \mathrm{C}$ for future use. PCR was performed using previously described primers (Thermo Fisher Scientific, Waltham, MA, United States) to amplify $\beta$-lactamase encoding genes from Ambler class A, bla $a_{\mathrm{PER}-1}$ (Yang et al., 2019); class B metallo- $\beta$-lactamases (MBLs), bla $a_{\mathrm{VIM}}$ (Ellington et al., 2007), bla $a_{\mathrm{NDM}}$ (Poirel et al., 2011); and class D oxacillinases, bla $a_{\text {OXA-23-like, bla }}$ OXA-24-like (Asadollahi et al., 2012), and bla $a_{\text {OXA-48-like }}$ (Poirel et al., 2011). Amplification conditions for class $\mathrm{D}$ oxacillinases genes were initial denaturation at $94^{\circ} \mathrm{C}$ for $5 \mathrm{~min}$; 35 cycles of denaturation at $94^{\circ} \mathrm{C}$ for $30 \mathrm{~s}$, annealing at $54^{\circ} \mathrm{C}$ for $30 \mathrm{~s}$, and elongation at $72^{\circ} \mathrm{C}$ for $30 \mathrm{~s}$; then a final extension at $72^{\circ} \mathrm{C}$ for $10 \mathrm{~min}$. Similar conditions were used to amplify bla $a_{\mathrm{PER}-1}$, but the annealing temperature was set at $50^{\circ} \mathrm{C}$ and elongation at $72^{\circ} \mathrm{C}$ for $60 \mathrm{~s}$. As for class B MBLs genes, an annealing at $58^{\circ} \mathrm{C}$ touchdown $52^{\circ} \mathrm{C}$ was implemented in a TECHNE thermocycler (Bibby Scientific Ltd., Staffordshire, United Kingdom). PCR assays for the detection of biofilm-related genes (bap, csuE, and ompA) were performed by a set of primers described in a previous study (Yang et al., 2019). Conditions of the PCR involved initial denaturation at $94^{\circ} \mathrm{C}$ for $5 \mathrm{~min}$, followed by 35 cycles of denaturation at $94^{\circ} \mathrm{C}$ for $60 \mathrm{~s}$, an annealing temperature at $57^{\circ} \mathrm{C}$ for $60 \mathrm{~s}$, and elongation at $72^{\circ} \mathrm{C}$ for $60 \mathrm{~s}$, and a final extension at $72^{\circ} \mathrm{C}$ for $10 \mathrm{~min}$. Gel electrophoresis was carried out using the ADVANCE electrophoresis system (Mupid-exu, Tokyo, Japan) at $5 \mathrm{~V} / \mathrm{cm}$ on $1.5 \%$ agarose gel, stained with $0.5 \mu \mathrm{g} / \mathrm{ml}$ ethidium bromide, and visualized under ultraviolet light.

\section{Antibacterial Activities of Cinnamic and Gallic Acids}

First, cinnamic and gallic acids (LOBA Chemie, Boisar, India) stock solutions were prepared (Daneshfar et al., 2008; Bradley et al., 2015). In brief, $1.5 \mathrm{~g}$ of cinnamic acid was dissolved in $10 \mathrm{ml}$ dimethyl sulfoxide (DMSO); then, distilled water was added to $100 \mathrm{ml}$, sonicated on water bath for $2 \mathrm{~h}$ at $80^{\circ} \mathrm{C}$, and $0.1 \mathrm{~N} \mathrm{NaOH}$ was added dropwise till complete solubilization, then distilled water was added to the final volume $(200 \mathrm{ml})$. For gallic acid, $4 \mathrm{~g}$ was dissolved in $150 \mathrm{ml}$ distilled water, sonicated on water bath for $30 \mathrm{~min}$; then distilled water was added to the final volume $(200 \mathrm{ml})$. Next, $100 \mu \mathrm{l}$ of cation adjusted Mueller Hinton broth CAMHB (Hi-Media, Mumbai, India) was dispensed in each well of a 96-well microtiter plate. Then, twofold serial dilutions of cinnamic or gallic acid solutions were prepared in the culture media at a final volume of $100 \mu \mathrm{l}$. Afterward, the wells were inoculated with $100 \mu \mathrm{l}$ of an overnight culture of the test isolate adjusted to $0.5 \mathrm{McF}$ arland standard, and plates were incubated at $37^{\circ} \mathrm{C}$ for $18-24 \mathrm{~h}$ (Bardbari et al., 2018). The positive control wells were the bacteria in CAMHB and the used solvent without cinnamic nor gallic acids. While wells without bacterial inoculum served as negative controls. The MICs of cinnamic and gallic acids were recorded as the lowest concentrations that totally inhibit visible bacterial growth.

\section{Antibiofilm Activities of Cinnamic and Gallic Acids at Sub-Inhibitory Concentrations}

Initially, $200 \mu \mathrm{l}$ of $0.5 \mathrm{McF}$ arland bacterial Suspensions were distributed in 96-well polystyrene microtiter plates. After that, $20 \mu \mathrm{l}$ cinnamic or gallic acid solutions were added to the wells at concentrations equivalent to $1 / 2$ and $1 / 4 \mathrm{MICs}$, and the plates were incubated for $24 \mathrm{~h}$ at $37^{\circ} \mathrm{C}$. Plates were washed twice with phosphate-buffered saline, stained with crystal violet solution $0.1 \% \mathrm{v} / \mathrm{v}$, and then the dye was resolubilized with $33 \% \mathrm{v} / \mathrm{v}$ acetic acid. The optical density of the wells was recorded at $630 \mathrm{~nm}$ using a microtiter plate reader (ELx800, Biotek, Winooski, VT, United States) (Tutar et al., 2016). Each assay was performed in triplicates, and wells free of dissolved cinnamic and gallic acids represented the positive controls for biofilm formation. The percentage of biofilm reduction (\%) was calculated as [(AcAs) $/ A c \times 100]$, where "Ac" is the $O D_{630}$ value of the positive control wells, and "As" is the $O D_{630}$ value of the cinnamic or gallic acids treated sample wells (Shao et al., 2015).

\section{Scanning Electron Microscopy Analysis of Antibiofilm Activities of Cinnamic and Gallic Acids}

A strong biofilm former A. baumannii isolate was selected to visualize the effect of cinnamic and gallic acids on its associated biofilm. First, $500 \mu \mathrm{l}$ of the bacterial suspension $\left(10^{8} \mathrm{CFU} / \mathrm{ml}\right)$ was dispensed into the wells of a 24 -well plate enclosing a round glass cover slip (13 mm, Menzel Gläser, Braunschweig, Germany). For treated wells, sub-MICs of cinnamic or gallic acids $(500 \mu \mathrm{l})$ were added and incubated for $24 \mathrm{~h}$ at $37^{\circ} \mathrm{C}$. Afterward, the formed biofilms on the coverslips were fixed in $2.5 \%$ glutaraldehyde for $30 \mathrm{~min}$ at room temperature in $0.1 \mathrm{M}$ cacodylate buffer ( $\mathrm{pH}$ 7.2). Subsequent post-fixation with osmium tetroxide and dehydration for $15 \mathrm{~min}$ with an ethanol gradient including 30,50, 70, 90, and 100\% v/v. The fixed biofilms were then coated with gold and examined with scanning electron microscope (JEOL JSM-6390LV, Tokyo, Japan) to compare treated isolates to untreated ones as a reference (Lemos et al., 2018).

\section{Growth Rate Analysis}

The growth rates of 10 strong biofilm formers, 10 weak biofilm formers, and ATCC19606 were measured. For each strain, $20 \mu \mathrm{l}$ of an overnight culture adjusted to $0.5 \mathrm{McF}$ arland standard were added to $180 \mu \mathrm{l}$ tryptic soy broth (TSB) in 96-well plates and incubated at $37^{\circ} \mathrm{C}$ for $24 \mathrm{~h}$. Bacterial growth was monitored by measuring the $O D_{600}$ in a microtiter plate reader (ELx800, Biotek, Winooski, VT, United States) every 4 h until 48 h. Results were recorded as means of absorbance readings from triplicate wells. In addition, the growth inhibitory activity of subinhibitory concentrations (1/2 and 1/4 MICs) of cinnamic and gallic acids was determined against these isolates. All determinations were 
performed as triplicates using untreated growth controls (Huang et al., 2020; Li et al., 2021).

\section{Statistical Analysis}

All analyses were carried out using $\mathrm{R}$ statistical platform ${ }^{1}$ in R-studio version 1.4.1106. Several R-packages were used in data analysis and visualization including readxl, ggplot2, polycor, and ggdendro. In quantitative variables, normality assumption was tested using chi-squared goodness-of-fit test. For normally distributed data, $t$-test and ANOVA were used to compare the means of two groups and multiple groups, respectively. KruskalWallis (KW) test was used to compare the medians for nonnormally distributed data. Mann-Whitney and Tukey's honestly significant difference (HSD) tests were applied as post-hoc tests using Bonferroni correction method for multiple comparisons in the Kruskal-Wallis and ANOVA tests, respectively. Fisher's exact (FE) test of independence was employed to analyze the associations between nominal variables. Moreover, the correlation between these variables was performed using the Spearman's rank correlation, and strength of the association was expressed as Spearman's correlation coefficient $\left(r_{\mathrm{s}}\right)$ between -1 and +1 . For all statistical analyses, $p<0.05$ were considered statistically significant.

\section{Ethical Approval}

All experiments and study protocols complied with relevant guidelines, regulations, and standards of the ethical committee of Faculty of Pharmacy, Ahram Canadian University, and international ethical guidelines for biomedical research. Since the collected A. baumannii isolates were obtained from biospecimen repositories at the Microbiology Laboratory in El-Demerdash Hospital, there was no direct or indirect contact with any patient, and consequently, no informed consent was required.

\section{RESULTS}

\section{Minimum Inhibitory Concentration Determination and Resistance Profiles}

Among the tested $A$. baumannii isolates $(n=90)$, resistance to levofloxacin $(n=81 ; 90 \%)$ was the most common, followed by resistance to amikacin $(n=65 ; 72.2 \%)$, imipenem $(n=62$; $68.9 \%)$, doxycycline $(n=60 ; 66.7 \%)$, and colistin $(n=11$; 12.2\%), as shown in Figures 1A,C. For additional exploration, we clustered the isolates based on their antibiotic susceptibility patterns, and the resultant clusters are presented as a heat map (Figure 1B). The details of the six clusters are presented in Figure 1D. Notably, clusters $1-3$ corresponded to $76.6 \%$ of the isolates revealing high prevalence of multiple drug resistance species. Only 4 out of 90 isolates were susceptible to the tested antibiotics exemplifying the high resistance rates detected in such isolates. High resistance level is usually described as having an MIC 16-fold or more the reported breakpoint (Mouton et al., 2018; Zhu et al., 2021). Accordingly MICs can be described as high because all the isolates have shown high resistance level

${ }^{1}$ https://www.r-project.org against levofloxacin ( $n=81 / 81$ ); three quarters have shown high resistance level against amikacin $(n=49 / 65), 63 \%$ against doxycycline $(n=38 / 60)$, 55\% against colistin $(n=6 / 11)$, and $34 \%$ against imipenem $(n=21 / 62)$. This is further demonstrated in the high MIC50 and MIC90 values of almost all the examined antibiotics (Table 1).

\section{Coexistence of Antimicrobial Resistance}

To evaluate the co-occurrence of resistance among various antibiotics, we built a correlation matrix between the tested agents employing the susceptibility patterns of the 90 isolates. As illustrated in Figure 2, positive and negative correlations were observed depending on the groups of antibiotics assessed. This analysis indicated that imipenem (IPM), amikacin (AMK), and levofloxacin (LVX) displayed the strongest correlations. Precisely, the Spearman's correlation coefficient $\left(r_{\mathrm{s}}\right)$ between AMK and IPM was 0.424 (FE test $p=0.00003$ ) and between AMK and LVX was 0.423 ( FE test $p=0.00003$ ). These results are in accordance with the recorded resistance patterns (Figure 1D) in which coresistance of AMK and IPM was detected in 54 isolates (60\%), while resistance to both AMK and LVX was documented in 66 isolates $(73 \%)$.

\section{Strength of Biofilm-Formation by A. baumannii Isolates and Growth Rate Analysis}

Effectively, the crystal violet biofilm assay differentiated A. baumannii isolates $(n=90)$ into strong, moderate, and weak biofilm formers. In particular, 11 isolates (12.2\%) were categorized as weak biofilm formers, 48 (53.3\%) were moderate, and $31(34.5 \%)$ had strong biofilm-forming abilities. No significant differences $(p>0.05)$ in growth rates of the strong and weak biofilm formers were observed (Supplementary Table 1), indicating that the difference in biofilm formation was not due to the growth rate.

\section{Relationship Between Antibiotic Susceptibility and Biofilm-Forming Ability}

The biofilm-forming abilities (strong, moderate, and weak) of $A$. baumannii isolates were determined among different antibiotic-resistance clusters (Table 2). Generally, a statistically significant association existed between clusters of isolates and intensity of biofilm ( $F E$ test $p=0.03$ ). For instance, strong biofilm formers were enriched in clusters I, II, and III with the respective percentages $9.7,41.9$, and $12.9 \%$. A similar tendency was observed for moderate biofilm formers with the percentages $4.2,31.1$, and $47.9 \%$. Since clusters I-III are MDR isolates, the differences between multidrug-resistant isolates and susceptible ones, based on their biofilm formation, were evaluated, and a significant association was attained (FE test $p=0.042$ ). To analyze whether the biofilm-forming ability is correlated with any of the tested antibiotics, we constructed a contingency table between the variables under consideration (Supplementary Table 2). 


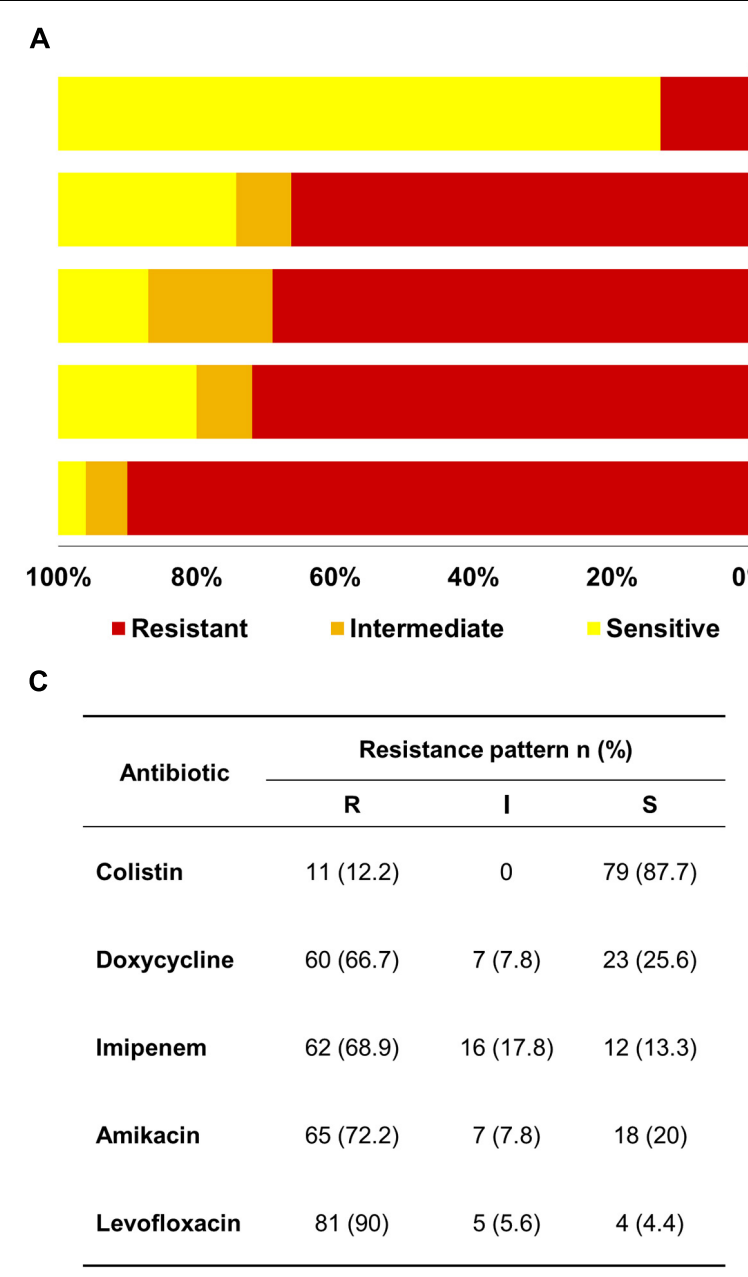

B

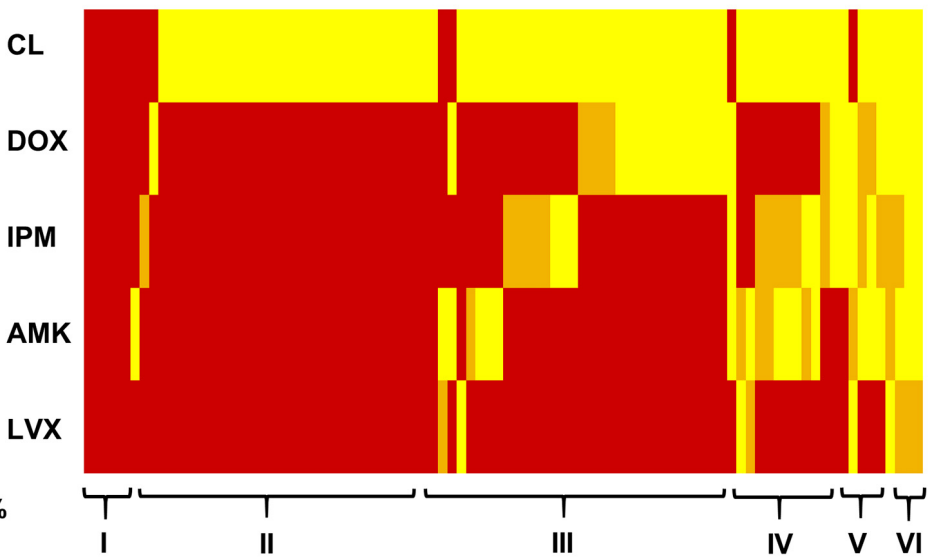

D

\begin{tabular}{|c|c|c|c|}
\hline Cluster & $\begin{array}{l}\text { No. of antibiotic } \\
\text { classes }\end{array}$ & $\begin{array}{l}\text { No. of isolates } \\
\text { (\%) }\end{array}$ & Resistance pattern \\
\hline $\mathbf{I}$ & 5 & $5(5.6)$ & CL, DOX, IPM, AMK, LVX \\
\hline \multirow[b]{2}{*}{ II } & \multirow[b]{2}{*}{4} & $30(33.3)$ & DOX, IPM, AMK, LVX \\
\hline & & $2(2.2)$ & CL, DOX, LVX, IPM or AMK \\
\hline \multirow{4}{*}{ III } & \multirow{4}{*}{3} & $17(18.9)$ & IPM, AMK, LVX \\
\hline & & $8(8.9)$ & DOX, AMK, LVX \\
\hline & & $5(5.5)$ & DOX, IPM, LVX or AMK \\
\hline & & $2(2.2)$ & CL, IPM, LVX or DOX \\
\hline \multirow{4}{*}{ IV } & \multirow{4}{*}{2} & $7(7.8)$ & DOX, LVX \\
\hline & & $2(2.2)$ & DOX, IPM \\
\hline & & $3(3.3)$ & AMK, LVX \\
\hline & & $1(1.1)$ & CL, LVX \\
\hline \multirow{2}{*}{$\mathbf{v}$} & \multirow{2}{*}{1} & $3(3.3)$ & LVX \\
\hline & & $1(1.1)$ & $\mathrm{CL}$ \\
\hline VI & - & $4(4.4)$ & None \\
\hline
\end{tabular}

FIGURE 1 | Sensitivity of $A$. baumannii isolates $(n=90)$ to different antibiotics as analyzed by MIC. (A) Stacked bar chart summarizing the resistance phenotypes of the 90 A. baumannii isolates to five antibiotics (y-axis). CL, colistin; DOX, doxycycline; IPM, imipenem; AMK, amikacin; LVX, levofloxacin. (B) Heatmap demonstrating the resistance pattern of each isolate as per the colors in the figure key (red, resistant; orange, intermediate; and yellow, sensitive). Isolates on the X-axis are sorted into six clusters footnoted on the heatmap. (C) Table summarizing the interpretation of MIC tests according to CLSI (2019). (D) Tabular representation of the detailed resistance patterns of the six clusters shown in Panel (B).

TABLE 1 | Distribution of Acinetobacter baumannii isolates ( $n=90)$ according to minimum inhibitory concentration (MIC) of the tested antimicrobial agents.

\begin{tabular}{|c|c|c|c|c|c|c|c|c|c|c|c|c|c|c|}
\hline \multirow[t]{2}{*}{ Antibiotic } & \multicolumn{14}{|c|}{ Minimum inhibitory concentration $(\mu \mathrm{g} / \mathrm{ml})$} \\
\hline & $<2$ & 2 & 4 & 8 & 16 & 32 & 64 & 128 & 256 & 512 & 1024 & $>1024$ & MIC50 & MIC90 \\
\hline Colistin & 39 & 40 & 0 & 2 & 3 & 0 & 2 & 1 & 0 & 2 & 0 & 1 & 2 & 8 \\
\hline Imipenem & 3 & 9 & 16 & 4 & 10 & 16 & 11 & 12 & 0 & 2 & 6 & 1 & 32 & 256 \\
\hline Doxycycline & 0 & 8 & 15 & 7 & 7 & 11 & 4 & 1 & 9 & 8 & 20 & 0 & 32 & 1024 \\
\hline Amikacin & 0 & 0 & 1 & 3 & 14 & 7 & 3 & 2 & 2 & 9 & 0 & 49 & $>1024$ & $>1024$ \\
\hline Levofloxacin & 0 & 4 & 5 & 3 & 8 & 28 & 11 & 28 & 3 & 0 & 0 & 0 & 32 & 128 \\
\hline
\end{tabular}

\section{Antibiotic-Resistance Correlations of Multidrug-Resistant A. baumannii Isolates}

Out of the 90 screened A. baumannii isolates, $30 \mathrm{MDR}$ isolates were selected for further study. None of the tested isolates were susceptible to levofloxacin, and over 90\% exhibited resistance to imipenem, doxycycline, and amikacin. On the other hand, only six (20\%) isolates displayed reduced susceptibility to colistin. A positive strong correlation between DOX and AMK resistance was observed $\left(r_{\mathrm{s}}=0.557, \mathrm{FE}\right.$ test $p=0.001)$. However, a moderate negative correlation between CL and AMK $\left(r_{\mathrm{s}}=-0.37, F E\right.$ test $\left.p=0.043\right)$ was found. 


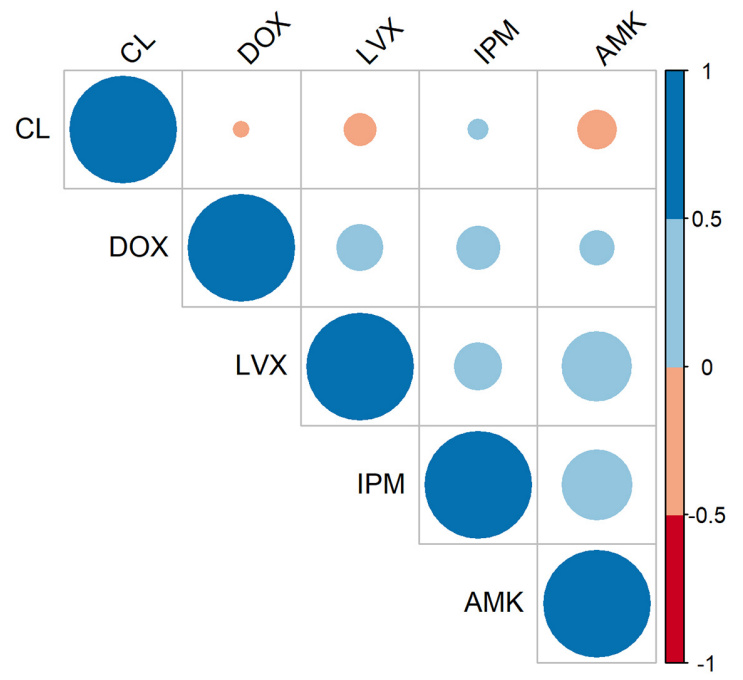

FIGURE 2 | Antibiotic-antibiotic correlations. Correlogram representing correlation coefficients between each pair of antibiotics according to the patterns of susceptibility of the A. baumannii 90 isolates. The color intensity represents Spearman's rank correlation coefficient $(r s)$ value (blue tones are positive correlations, and red tones are negative correlations).

\section{Molecular Detection of $\beta$-Lactamase-Encoding Genes and Genotypic-Phenotypic Correlations}

Results of the detected $\beta$-lactamase-encoding genes and the corresponding imipenem MIC distributions for the $30 \mathrm{MDR}$ A. baumannii are shown in Table 3. The results revealed almost equal occurrence of ambler class B MBLs $(n=13)$, ambler class $\mathrm{D}$ serine $\beta$-lactamases $(n=12)$, and class A $\beta$-lactamases $(n=14)$. Nevertheless, none of $A$. baumannii isolates harbored blaXA-24-like and $b l a_{\text {OXA-48-like }}$ genes. Interestingly, a strong negative correlation was observed between MBLs and serine $\beta$-lactamases genes $\left(r_{\mathrm{s}}=-0.714, F E\right.$ test $\left.p=0.0001\right)$. On the contrary, blaviM gene positively correlated with bla $a_{\mathrm{NDM}}$ $\left(r_{s}=0.398, F E\right.$ test $\left.p=0.029\right)$. Furthermore, the effects of different genotypes on imipenem resistance of the isolates were assessed. A Kruskal-Wallis test showed that MBLs and class A $\beta$-lactamases genes significantly affect the imipenem

TABLE 2 | Distribution of biofilm forming-abilities of Acinetobacter baumannii isolates among different antibiotic-resistance clusters.

\begin{tabular}{lccc}
\hline \multirow{2}{*}{$\begin{array}{l}\text { Cluster } \\
\text { Classes) }\end{array}$} & \multicolumn{3}{c}{ Biofilm formation $\boldsymbol{n}$ (\% column) } \\
\cline { 2 - 4 } & $\begin{array}{c}\text { Strong } \\
(\boldsymbol{n}=\mathbf{3 1 )}\end{array}$ & $\begin{array}{c}\text { Moderate } \\
(\boldsymbol{n}=\mathbf{4 8})\end{array}$ & $\begin{array}{c}\text { Weak } \\
(\boldsymbol{n}=\mathbf{1 1})\end{array}$ \\
\hline I (5) & $3(9.7 \%)$ & $2(4.2 \%)$ & $0(0 \%)$ \\
II (4) & $13(41.9 \%)$ & $15(31.3 \%)$ & $4(36.4 \%)$ \\
III (3) & $4(12.9 \%)$ & $23(47.9 \%)$ & $5(45.5 \%)$ \\
IV (2) & $5(16.1 \%)$ & $7(14.6 \%)$ & $1(9.1 \%)$ \\
V (1) & $3(9.7 \%)$ & $1(2.1 \%)$ & $0(0 \%)$ \\
VI (0) & $3(9.7 \%)$ & $0(0 \%)$ & $1(9.1 \%)$
\end{tabular}

MICs, $H(2)=6.0504, p=0.0485$. The difference in imipenem MICs between a genotype of a single MBL and a genotype of MBL combined with class A $\beta$-lactamases was statistically significant (post-hoc Mann-Whitney test, $p=0.02668$ ). On the other hand, the difference in imipenem MICs was not significant among genotypes belonging to class $\mathrm{D}$ and class $\mathrm{A}$ $\beta$-lactamases $(p>0.05)$. Moreover, isolates harboring resistance genes were enriched in strong and moderate biofilm formers (Supplementary Table 3), but only bla $a_{\mathrm{VIM}}$ and $b l a_{\mathrm{OXA}-23}$ genes were significantly associated with the biofilm-forming ability (FE test, $p=0.0347)$.

\section{Correlation Between Biofilm-Formation Ability and Detection of Biofilm-Related Genes}

The incidence of bla $a_{\mathrm{PER}-1}$, bap, ompA, and $c s u \mathrm{E}$ genes amid the isolates was 46.7, 83.8, 76.7, and 90\%, respectively. The tested isolates were classified based on the patterns of genes distribution using the unweighted pair group method with arithmetic mean (UPGMA) algorithm for hierarchical clustering (Figure 3). As shown in the dendrogram (Figure 3A), strong biofilm-forming isolates occupied a single major clade. However, a few moderate and weak biofilm formers were clustered in close vicinity of the strong ones. After analyzing the association between the biofilm-forming ability and biofilm-related genes, a statistically significant difference was found with ompA (FE test $p=0.0125)$, $o m p \mathrm{~A}+b a p(F E$ test $p=0.0122), o m p \mathrm{~A}+\operatorname{csu} \mathrm{E}(F E$ test $p=0.02)$, and $o m p \mathrm{~A}+b a p+c s u \mathrm{E}(F E$ test $p=0.02)$. Furthermore, a strong positive correlation existed between $c s u E$ along with both bap and ompA, genes ( $r_{\mathrm{s}}=0.745$ and 0.604 , respectively, FE test $p<0.05$ ). Similarly, bap positively correlated with ompA $\left(r_{\mathrm{s}}=0.599\right.$, FE test $p=0.00047)$. Intriguingly, the concurrence of either ompA and bap or ompA and $c s u \mathrm{E}$ had equivalent predictive value of strong biofilm formation $(100 \%)$ as the simultaneous detection of the three genes ompA, bap, and $c s u \mathrm{E}$ (Table 4).

\section{Antimicrobial Activity of Gallic and Cinnamic Acids}

The results revealed a promising antimicrobial activity against A. baumannii; MICs of cinnamic acid ranged from 1.01 to $1.67 \mathrm{mg} / \mathrm{ml}$, while those of gallic acid ranged from 1.32 to $2.11 \mathrm{mg} / \mathrm{ml}$ (Figure 4). Cinnamic acid (mean MICs $=1.2 \mathrm{mg} / \mathrm{ml}$ ) displayed higher antibacterial activity against $A$. baumannii as compared to that of gallic acid (mean MICs $=1.65 \mathrm{mg} / \mathrm{ml}$ ) $\left(t\right.$-test $\left.=-10.947, p=8.147 \times 10^{-12}\right)$.

\section{The Anti-biofilm Activities of Gallic and Cinnamic Acids}

Notably, both gallic and cinnamic acids resulted in significant reductions in the biofilm formation associated with $A$. baumannii isolates (Figure 5). The superlative antibiofilm activity was attained at $1 / 2$ MIC, which led to inhibition percentages reaching 82 and $91 \%$ for cinnamic and gallic acids, respectively. A statistically significant difference in the effect between the subinhibitory concentrations of cinnamic and gallic acids was observed by one-way ANOVA $[F(3)=3.415, p=0.0198]$. For 
TABLE 3 | Prevalence of $\beta$-lactamase-encoding genes with the corresponding imipenem minimum inhibitory concentration (MIC) distributions for 30 isolates of Acinetobacter baumannii.

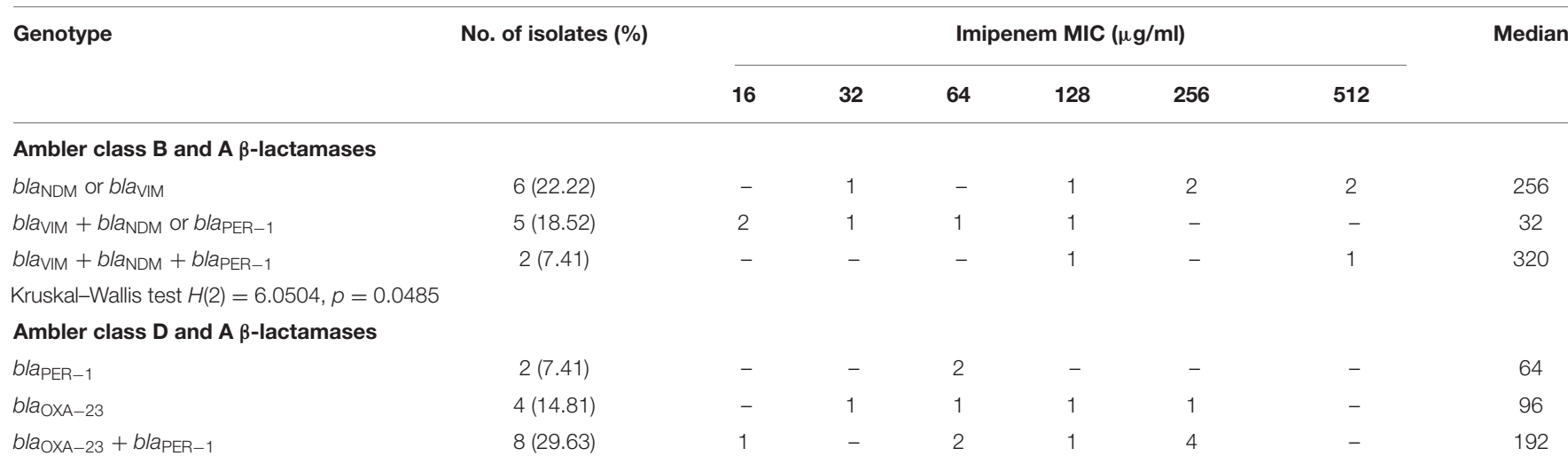

Kruskal-Wallis test $H(2)=1.3247, p=0.5156$

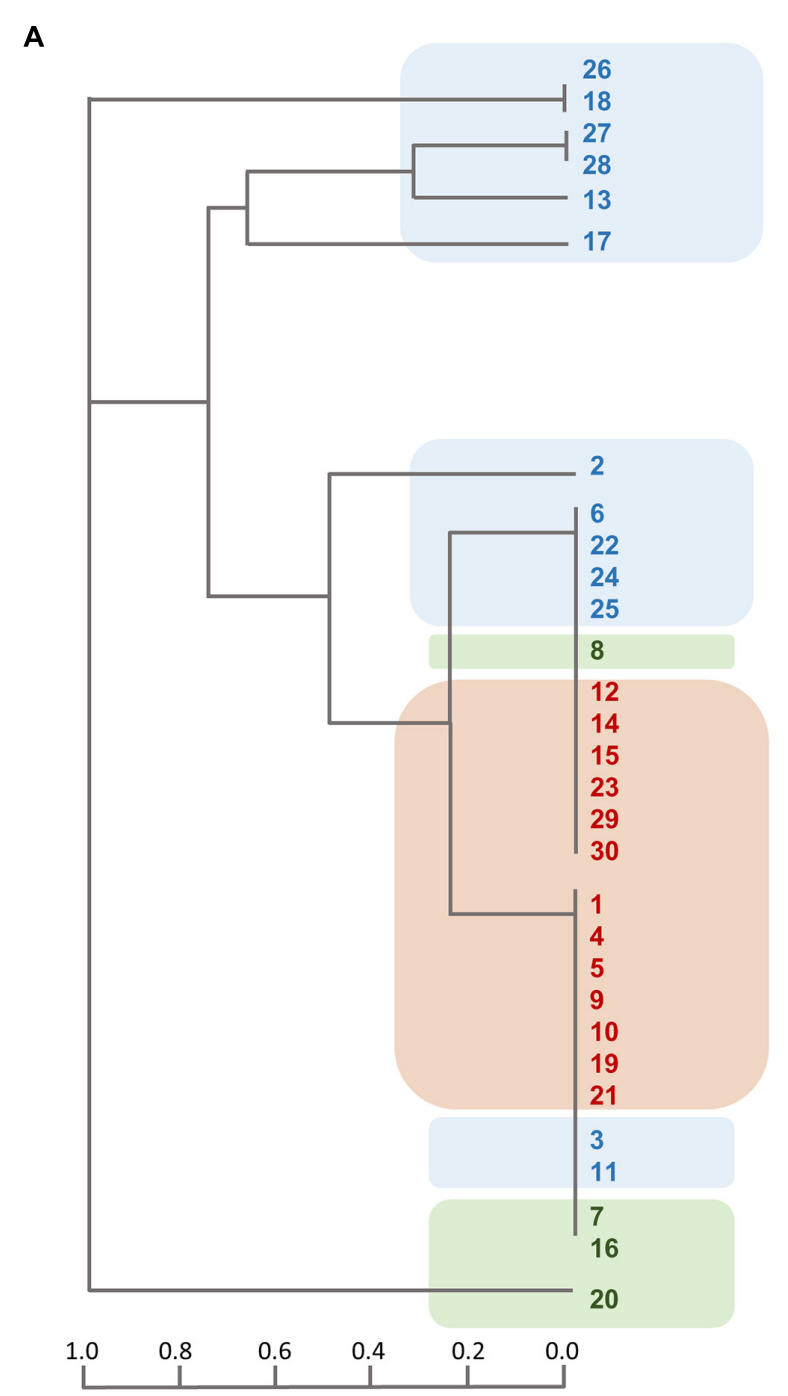

B

\begin{tabular}{|c|c|c|c|c|c|c|}
\hline Isolate No. & bap & $\operatorname{csu} \mathrm{E}$ & ompA & $b / a_{\text {PER }}$ & $\begin{array}{l}\text { Biofilm } \\
\text { strength }\end{array}$ & Mean $O_{630}$ \\
\hline Ac26 & - & - & - & + & Moderate & 1.16 \\
\hline Ac18 & - & - & - & + & Moderate & 0.925 \\
\hline Ac27 & + & + & - & - & Moderate & 0.842 \\
\hline Ac28 & + & + & - & - & Moderate & 0.742 \\
\hline Ac13 & + & + & - & + & Moderate & 0.742 \\
\hline Ac17 & - & + & - & - & Moderate & 1.251 \\
\hline Ac2 & - & + & + & - & Moderate & 1.224 \\
\hline Ac6 & + & + & + & - & Moderate & 1.029 \\
\hline Ac22 & + & + & + & - & Moderate & 0.941 \\
\hline Ac24 & + & + & + & - & Moderate & 1.086 \\
\hline Ac25 & + & + & + & - & Moderate & 1.242 \\
\hline Ac8 & + & + & + & - & Weak & 0.612 \\
\hline Ac12 & + & + & + & - & Strong & 1.521 \\
\hline Ac14 & + & + & + & - & Strong & 1.422 \\
\hline Ac15 & + & + & + & - & Strong & 1.63 \\
\hline Ac23 & + & + & + & - & Strong & 1.409 \\
\hline Ac29 & + & + & + & - & Strong & 1.567 \\
\hline Ac30 & + & + & + & - & Strong & 1.667 \\
\hline Ac1 & + & + & + & + & Strong & 1.357 \\
\hline Ac4 & + & + & + & + & Strong & 1.691 \\
\hline Ac5 & + & + & + & + & Strong & 1.451 \\
\hline Ac9 & + & + & + & + & Strong & 1.403 \\
\hline Ac10 & + & + & + & + & Strong & 1.374 \\
\hline Ac19 & + & + & + & + & Strong & 1.703 \\
\hline Ac21 & + & + & + & + & Strong & 1.32 \\
\hline Ac3 & + & + & + & + & Moderate & 1.125 \\
\hline Ac11 & + & + & + & + & Moderate & 1.113 \\
\hline Ac7 & + & + & + & + & Weak & 0.541 \\
\hline Ac16 & + & + & + & + & Weak & 0.431 \\
\hline Ac20 & - & - & - & - & Weak & 0.521 \\
\hline
\end{tabular}

FIGURE 3 | (A) Dendrogram signifying the clustering relatedness of 30 MDR A. baumannii isolates based on their PCR results for biofilm-related genes. The clusters are highlighted according to their biofilm strength (brown, strong; blue, moderate; green, weak biofilm forming). (B) Tabular presentation of the PCR screening results of biofilm associated genes and corresponding mean $O D_{630}$ values. 
TABLE 4 | Genotype-phenotype associations of biofilm-related genes with biofilm formation.

\begin{tabular}{|c|c|c|c|c|}
\hline \multirow[t]{2}{*}{ Biofilm-related genes } & \multicolumn{3}{|c|}{$\begin{array}{l}\text { Biofilm formation } n \\
\text { ( } \% \text { column) }\end{array}$} & \multirow[t]{2}{*}{$\begin{array}{l}\text { Fisher's } \\
\text { exact test }\end{array}$} \\
\hline & Strong & Moderate & Weak & \\
\hline OmpA + bap & & & & $p=0.012$ \\
\hline Both negative & $0(0 \%)$ & $3(23 \%)$ & $1(25 \%)$ & \\
\hline Only one positive & $0(0 \%)$ & $4(31 \%)$ & $0(0 \%)$ & \\
\hline Both positive & $13(100 \%)$ & $6(46 \%)$ & $3(75 \%)$ & \\
\hline OmpA + csuE & & & & $p=0.02$ \\
\hline Both negative & $0(0 \%)$ & $2(15.5 \%)$ & $1(25 \%)$ & \\
\hline Only one positive & $0(0 \%)$ & $4(31 \%)$ & $0(0 \%)$ & \\
\hline Both positive & $13(100 \%)$ & $7(53.5 \%)$ & $3(75 \%)$ & \\
\hline OmpA + bap + csuE & & & & $p=0.02$ \\
\hline All negative & $0(0 \%)$ & $2(15.5 \%)$ & $1(25 \%)$ & \\
\hline Only one positive & $0(0 \%)$ & $1(7.5 \%)$ & $0(0 \%)$ & \\
\hline Only two positive & $0(0 \%)$ & $4(31 \%)$ & $0(0 \%)$ & \\
\hline All positive & $13(100 \%)$ & $6(46 \%)$ & $3(75 \%)$ & \\
\hline OmpA + bap + bla $a_{\mathrm{PER}-1}$ & & & & $p=0.027$ \\
\hline All negative & $0(0 \%)$ & $1(7.5 \%)$ & $1(25 \%)$ & \\
\hline Only one Positive & $0(0 \%)$ & 5 (38.5\%) & $0(0 \%)$ & \\
\hline Only two Positive & $6(47 \%)$ & $5(38.5 \%)$ & $1(25 \%)$ & \\
\hline All Positive & $7(53 \%)$ & $2(15.5 \%)$ & $2(50 \%)$ & \\
\hline OmpA $+c s u E+b l a_{\mathrm{PER}-1}$ & & & & $p=0.015$ \\
\hline All negative & $0(0 \%)$ & $0(0 \%)$ & $1(25 \%)$ & \\
\hline Only one positive & $0(0 \%)$ & $5(38.5 \%)$ & $0(0 \%)$ & \\
\hline Only two positive & $6(47 \%)$ & $6(46 \%)$ & $1(25 \%)$ & \\
\hline All positive & $7(53 \%)$ & $2(15.5 \%)$ & $2(50 \%)$ & \\
\hline
\end{tabular}

a deeper investigation of the antibiofilm activities of both acids, we reanalyzed their effects after dissecting the isolates into strong, moderate, and weak biofilm formers (Figure 6). Regarding strong biofilm formers (Figure 6A), subinhibitory concentrations of gallic acid displayed enhanced effect against all isolates except Ac9, Ac12, Ac15, and Ac29 ( $r s=0.598, p<0.05$ ). Conversely, subinhibitory concentrations of cinnamic acid showed greater activity on all moderate biofilm formers, except Ac2, Ac3, Ac17, Ac22, and Ac24 (Figure 6B). A similar trend was observed in weak biofilm formers (Figure 6C). Statistical analysis showed that the type and concentration of the tested agent significantly affected reduction in biofilm formation among strong biofilm formers $[F(3,36)=6.4152, p<0.05]$. As shown in Figure 7A, a higher antibiofilm activity of the subinhibitory concentrations $(1 / 2$ and $1 / 4 \mathrm{MIC})$ of gallic acid (mean reduction $=73.70$ and $68.25 \%$, respectively) was observed as compared to cinnamic acid (mean reduction $=64.93$ and 58.59\%, respectively), whereas analysis of moderate biofilm forming isolates proved enhanced activity of the subinhibitory concentrations $(1 / 2$ and $1 / 4 \mathrm{MIC}$ ) of cinnamic acid (mean reduction $=65.70$ and $59.63 \%$, respectively) versus gallic acid (mean reduction $=62.02$ and $48.65 \%$, respectively) $[F(3,36)=6.5257, p<0.05]$ (Figure 7B). Concerning weak biofilm formers, despite the large discrepancies between the effect of subinhibitory concentrations (1/2 and 1/4 MIC) of cinnamic acid (mean reduction $=69.20$ and $61.13 \%$, respectively) versus gallic acid (mean reduction $=54.11$ and

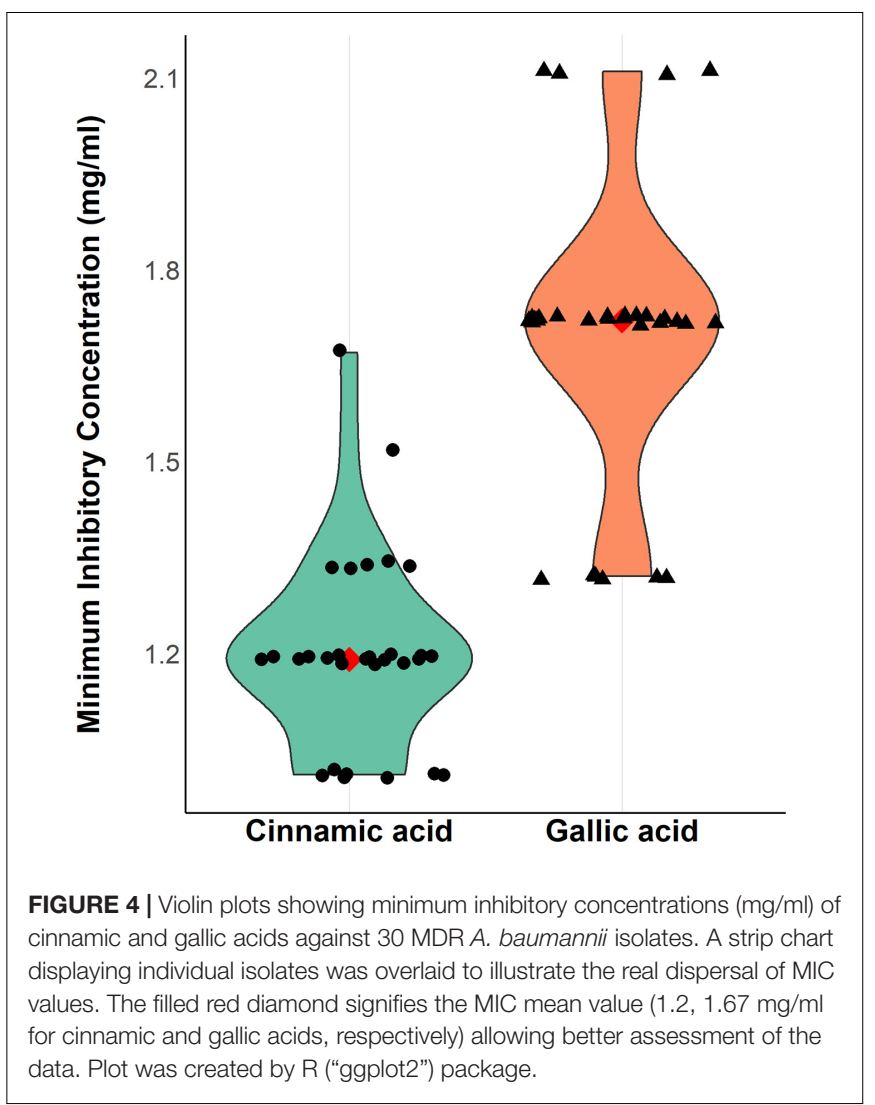

$47.64 \%$, respectively), no statistically significant difference was detected $(p>0.05)$, which may be attributed to small number of isolates in this group (Figure 7C). Noteworthy, the effect of subinhibitory concentrations (1/2 and 1/4 MICs) of cinnamic and gallic acids on the growth of the tested $A$. baumannii isolates are shown in figures (Supplementary Figures 1,2). Despite the slight growth pattern differences between the control and some of the treated isolates, sub-MICs of cinnamic and gallic acids generally did not affect the viability of the tested strains.

\section{Impact of Resistance Profiles and Biofilm-Related Genes on Biofilm Susceptibility to Gallic and Cinnamic Acids}

The only statistically significant positive correlation between the antibiotic MICs and the percentages of biofilm reduction was shown with levofloxacin $\left(r_{\mathrm{s}}=0.45, F E\right.$ test $\left.p=0.0115\right)$. As for the plausible effect of biofilm-related genes, including bap, csuE, ompA, and bla $a_{\mathrm{PER}-1}$, on the documented percentages of biofilm reduction, a statistically significant difference was obtained using sub-MICs of gallic acid $[F(7)=3.293, p=0.015]$ within the following genotype pairs, $\left(b a p+c s u \mathrm{E}+\right.$ ompA + bla $_{\mathrm{PER}-1}$ and $\left.b l a_{\mathrm{PER}-1}\right)($ means $=71.8$ vs. $44.6 \%)$ and (bap $+c s u \mathrm{E}+o m p \mathrm{~A}+b \operatorname{la}_{\mathrm{PER}-1}$ and no genes) (means $=71.8$ vs. $36.34 \%$ ). 


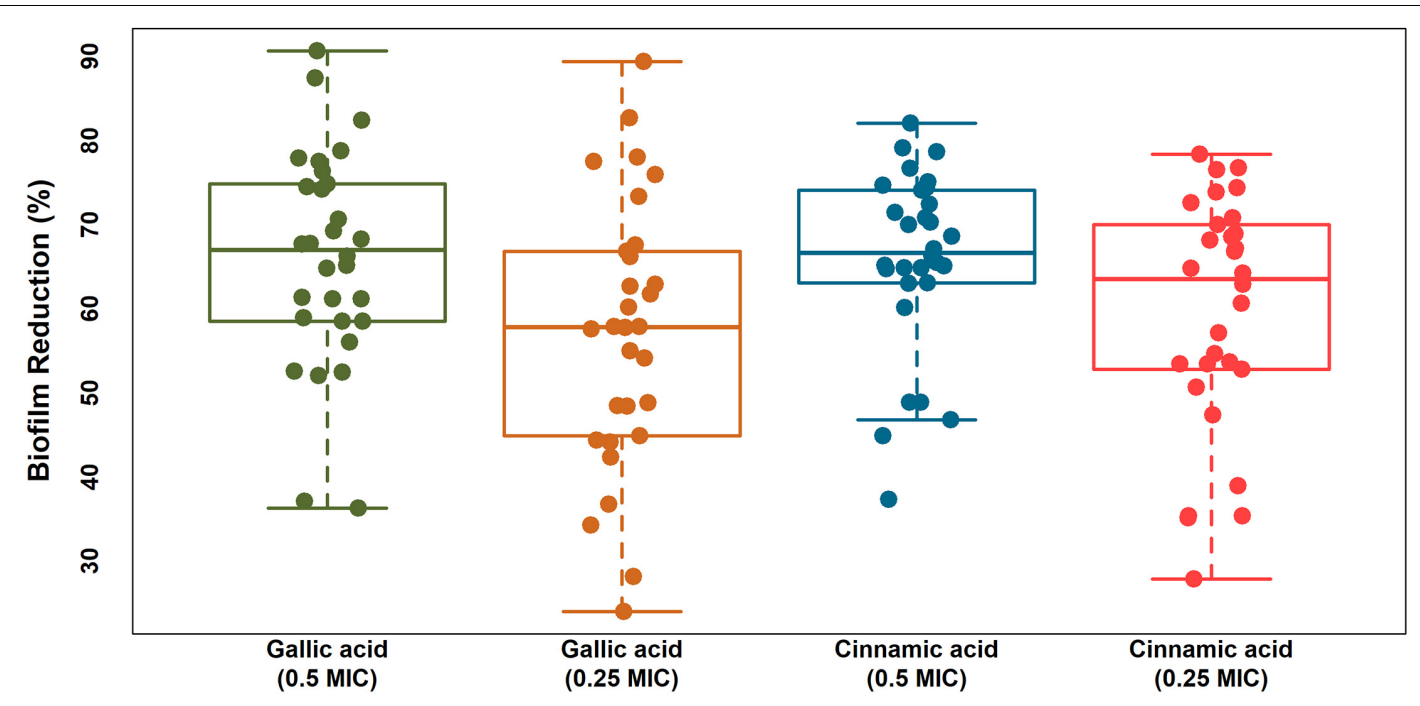

FIGURE 5 | Boxplots displaying biofilm reduction (\%) caused by subinhibitory concentrations (1/2 and 1/4 MICs) of cinnamic and gallic acids. A strip chart displaying individual isolates was overlaid to illustrate the real dispersal of reduction percentages. The means were significantly different [ANOVA $F(3), p=0.0198]$. Plot was created by R ("ggplot2") package.

\section{SEM Analysis of Antibiofilm Activities of Cinnamic and Gallic Acids}

SEM images of the untreated control biofilm showed a dense layer of colonized cells (Figure 8A), whereas a conspicuous inhibition of A. baumannii biofilm formation was noticed in the treated biofilm in which cells were individually visualized (Figures 8B,C). It is noteworthy that gallic acid possessed significantly higher activity (Figure $\mathbf{8 C}$ ) compared to cinnamic acid (Figure 8B), consistent with our findings using microtiter plate assay.

\section{DISCUSSION}

A. baumannii infections pose a serious concern triggered by the elevated rates of multidrug resistance (Eze et al., 2018). This problem is further aggravated by its ability to form biofilms (Yang et al., 2019). Antibiotic resistance in biofilms is estimated to be $\sim 1,000$-fold higher than in planktonic cells, thus restricting available choices of effective antimicrobial treatments (Hall and Mah, 2017). In the present study, 76.6\% (69/90) of our isolates were MDR displaying resistance patterns consistent with previous reports (Tchuinte et al., 2019; Araújo Lima et al., 2020). Our findings reveal higher levels of colistin resistance (12.2\%) compared to recent studies from the region (Abdulzahra et al., 2018; Elham and Fawzia, 2019). This noticeable increase in colistin resistance is alarming because it is considered the last resort antibiotic for the treatment of severe infections caused by carbapenem resistant A. baumannii (Thet et al., 2020). Moreover, we found statistically significant correlations between resistance to AMK and to both IPM and LVX ( $r$ s $=0.424$ and 0.423 , respectively). Yu et al. (2007) previously reported a widespread occurrence of amikacin resistance in imipenemresistant $A$. baumannii due to aminoglycoside resistance methyltransferase (ArmA). Similar correlations between IPM and LVX were recently described (El-Din et al., 2021), this may be attributed to the various impacts that resulted from a mutation at one or a few genetic loci (Adamus-Bialek et al., 2013). Our isolates exhibited much higher frequency $(100 \%)$ of biofilm production as opposed to A. baumannii clinical isolates (70.1\%) lately investigated from Egypt (Asaad et al., 2021), Iran (70.6\%) (Ranjbar and Farahani, 2019), and China (54\%) (Chen et al., 2020). Several studies linked high frequency of biofilm formation in A. baumannii with extended survival and resistance to external stresses such as limited nutrients and dehydration (Badave and Kulkarni, 2015; Sung, 2018). The nature of association between resistance profiles and biofilm formation in A. baumannii is somewhat controversial (Eze et al., 2018). According to some studies, biofilm formation is strongly associated with MDR strains than with susceptible ones (Babapour et al., 2016; Bardbari et al., 2017; Yang et al., 2019). On the other hand, an inverse relation between biofilm formation and antibiotic resistance has been lately documented (Qi et al., 2016; Shenkutie et al., 2020). The current study investigated the potential association between phenotypic-genotypic resistance profiles and the biofilm-forming ability of clinical A. baumannii isolates. In view of this, our results highlight a significant association between MDR and biofilm formation. Relevant finding of this association was proclaimed by researchers from Egypt (Asaad et al., 2021), India (Badave and Kulkarni, 2015), Bangladesh (Nahar et al., 2013), Iran (Ranjbar and Farahani, 2019), and Mexico (Bocanegra-Ibarias et al., 2015). Increased synthesis of exopolysaccharide in biofilm-forming A. baumannii probably creates a barrier to antibiotic penetration, hence development of resistance. Slow growth rate, variations in the cellular physiology of cells, and enhanced horizontal gene transfer within the biofilm communities may be another explanation for high resistance associated to biofilms (Lewis, 2001; Donlan and Costerton, 2002; 


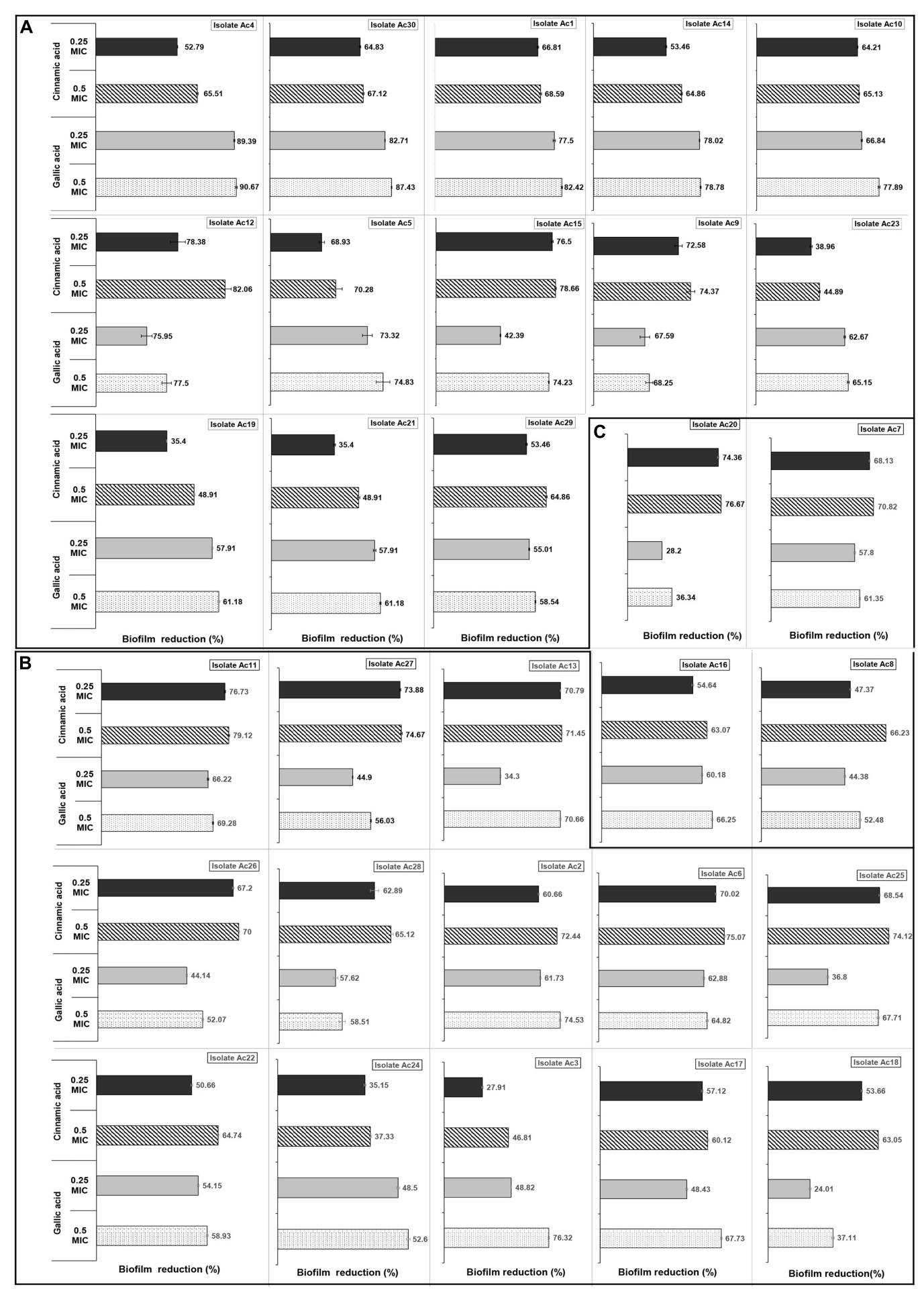

FIGURE 6 | Inhibitory effect of cinnamic and gallic acids on biofilm formation of each of the 30 MDR A. baumannii isolates. Clustered bar charts demonstrate biofilm reduction (\%) following treatment with subinhibitory concentrations of cinnamic acid 1/2 MICs (stripped bars), 1/4 MICs (black bars), and gallic acid 1/2 MICs (dotted bars), and 1/4 MICs (gray bars) on (A) strong, (B) moderate, and (C) weak biofilm formers.

Yang et al., 2019). Albeit not every resistance profile of our isolates was associated with stronger biofilm formation, a statistically significant correlation occurred with susceptibility to certain antibiotics. On the one hand, biofilm formation was associated $(p<0.05)$ with resistance to amikacin. A recent study (Yang et al., 2019) reported the association between resistance to 
A

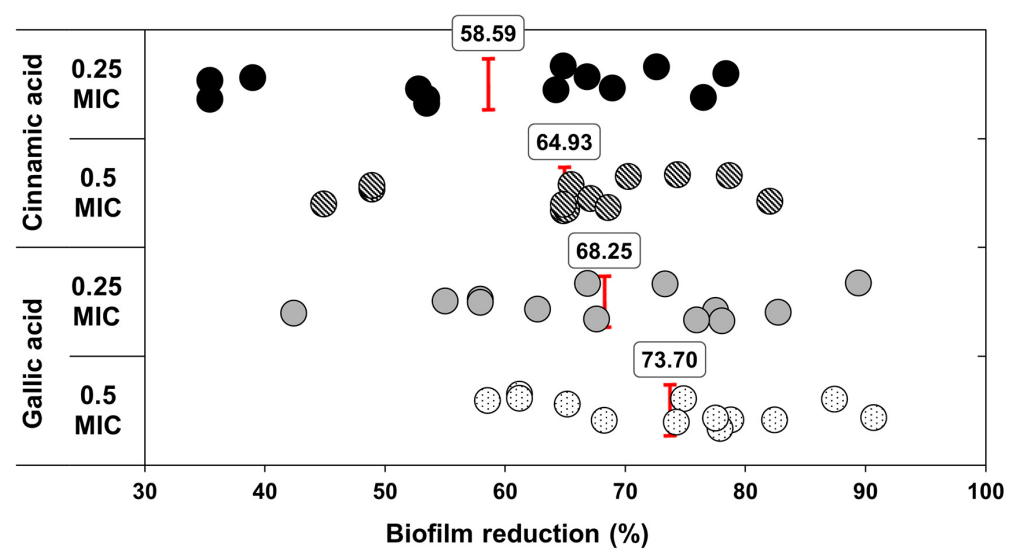

B

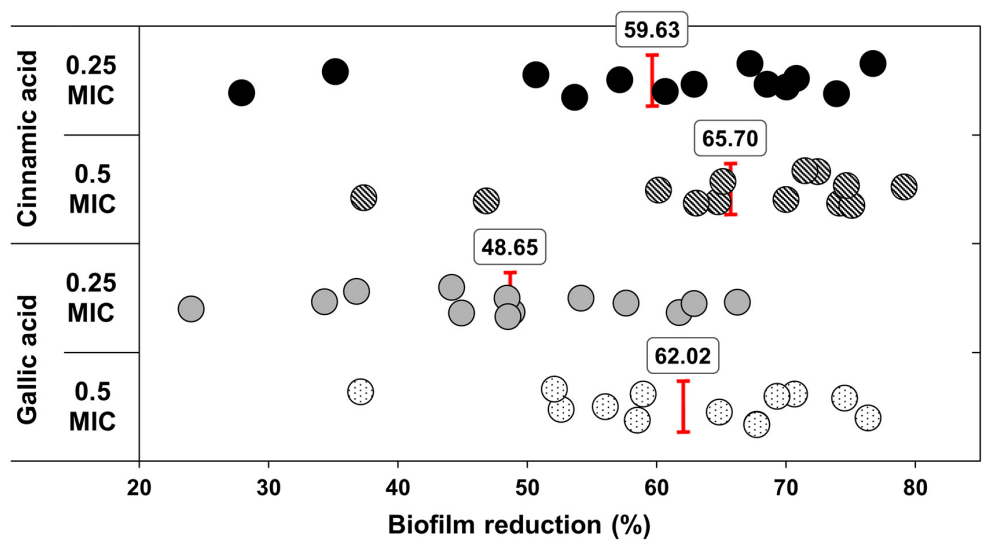

C

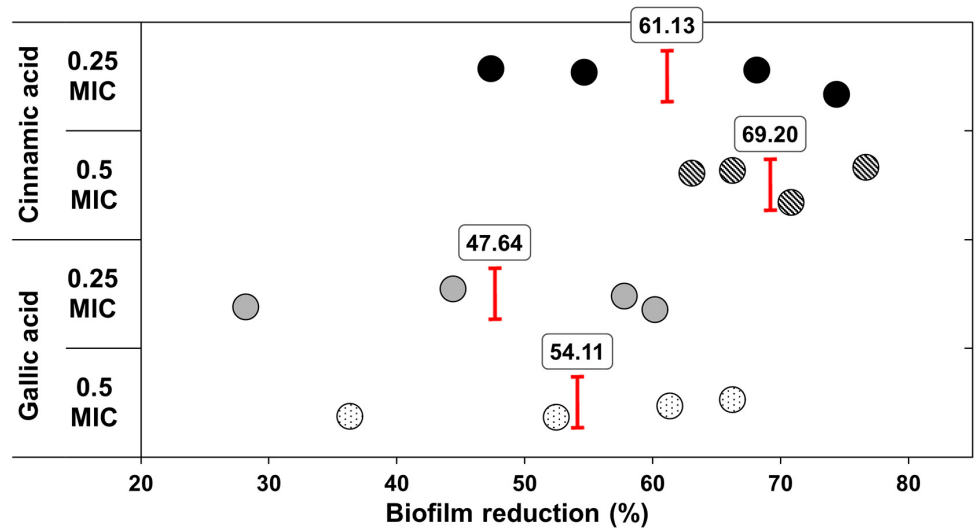

FIGURE 7 | Antibiofilm activities of sub-MICs of cinnamic and gallic acids. Scatter plot representing biofilm reduction (\%) for each sample. Mean reduction percentages of $1 / 2$ and $1 / 4 \mathrm{MICs}$ cinnamic and gallic acids are illustrated against (A) strong biofilm formers [ANOVA $F(3)=6.4152, p<0.05]$, (B) moderate biofilm formers [ANOVA $F(3)=6.5257, p<0.05$ ], and (C) weak biofilm formers [ANOVA $F(3)=2.3125, p>0.05$ ].

aminoglycosides and biofilm formation. This might be explained by induced gene regulation offering a fitness advantage for such resistant strains to form biofilms (Qi et al., 2016). We also noted a significant relation between biofilm formation and levofloxacin resistance in agreement with other studies (Coyne et al., 2010; He et al., 2015) reporting overexpression of the AdeFGH efflux pump leading to high-level resistance to fluoroquinolone and increased biofilm formation.
Several Ambler class $\beta$-lactamase-encoding genes were detected, bla $a_{\mathrm{OXA}-23}(40 \%), b l a_{\mathrm{VIM}}(36.6 \%)$, and $b l a_{\mathrm{NDM}}$

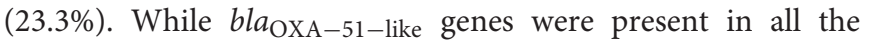
isolates, $b l a_{\mathrm{OXA}}-24^{-}$and $b l a_{\mathrm{OXA}}-48^{-e n c o d i n g}$ genes were absent. Acquired $\beta$-lactamase-encoding genes, disseminating via mobile genetic elements, were concurrently present in $50 \%$ of the isolates, regrettably warning for further spread via horizontal gene transfer (Linciano et al., 2020). Although MBLs are 

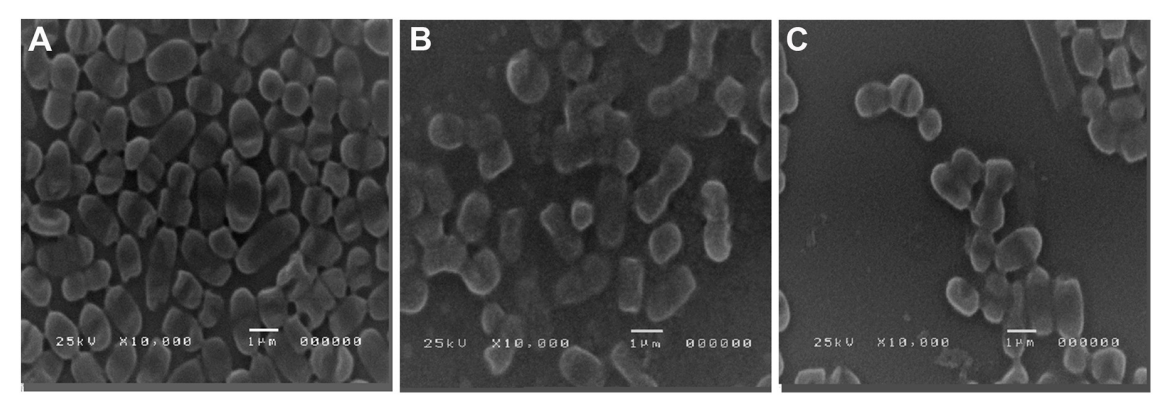

FIGURE 8 | Scanning electron microscopy images of (A) untreated strong biofilm-former $A$. baumannii isolate, after (B) a 24-h treatment with sub-MIC cinnamic acid, and (C) a 24-h treatment with sub-MIC gallic acid. Magnification 10,000x.

usually coexpressed with serine $\beta$-lactamases (Meini et al., 2014), we conversely delineate a negative correlation between their encoding genes $\left(r_{\mathrm{s}}=-0.714, p<0.05\right)$. Noteworthy, the coexistence of bla $a_{\mathrm{PER}-1}$ with multiple MBL encoding genes was significantly associated with high imipenem MICs. Likewise, researchers from Iran (Bagheri Josheghani et al., 2015) and Saudi Arabia (Aly et al., 2016) characterized the high prevalence of bla $a_{\mathrm{PER}-1}$ gene among carbapenem-resistant A. baumannii.

Another goal of this study was to assess the relationships between biofilm formation and the presence of biofilm-related and antibiotic resistance genes in the isolates. For this purpose, we found a significant association between biofilm formation and the presence of either bla OXA-23 or bla VIM genes $(p<0.05)$. Formerly, Azizi et al. (2015) reported that A. baumannii isolates with class-D OXA carbapenemases genes had strong biofilm capability. Several studies showed that Pseudomonas aeruginosa isolates harboring bla $a_{\mathrm{VIM}}$ gene produced stronger biofilms (Heydari and Eftekhar, 2015). To the best of our knowledge, this is the first report of association between biofilm formation and bla $a_{\mathrm{VIM}}$ gene in A. baumannii. This may represent a profound concern due to the likelihood of horizontal gene transfer between isolates closely located within biofilms (Abe et al., 2020; Uruén et al., 2021). Regarding the biofilm-related genes, our results agree with that of Zeighami et al. (2019), in which the most frequently detected gene was csu $\mathrm{E}(90 \%)$, followed by bap (83.8\%), ompA (76.7\%), and bla $a_{\mathrm{PER}-1}(46.7 \%)$. In compliance with other investigators (Bardbari et al., 2017), no relationship between biofilm formation and production of bla $a_{\text {PER-1 }}$ could be established. A conceivable rationalization for this could be that bla $a_{\mathrm{PER}-1}$ gene might be associated with increased cell adhesiveness without promoting biofilm formation (Yang et al., 2019). In A. baumannii isolates, ompA is involved in resistance to antimicrobial agents, needed for attachment to eukaryotic cells, and partially contributes to biofilm formation (Yang et al., 2019). This investigation validated a significant association between the biofilm intensity and presence of ompA gene $(p=0.0125)$. Remarkably, we recorded a strong positive correlation between ompA and both bap, which is responsible for the expression of biofilm-associated proteins on the bacterial cell surfaces, and $c s u \mathrm{E}$, which mediates adhesion and biofilm formation. Moreover, a statistically significant association was detected between these genes and the biofilmforming strength. Hence, these gene pairs are considered of great diagnostic value for the discrimination of strong biofilmforming phenotype. Strikingly, three of the weak biofilm-forming isolates also had some biofilm-related genes, emphasizing the role of factors such as stress, nutrition, environment, and pathogen itself in biofilm induction and development (Al-Shamiri et al., 2021).

Taken together, the above analyses disclose the scale of the problem imposed by MDR A. baumannii. During the last decade, research studies on remedies with natural products were conducted to provide alternative antimicrobials for combating MDR isolates. For instance, cinnamic acid displayed noticeable antimicrobial effects against food-borne pathogens and standard and clinical isolates (Alves et al., 2013; Guzman, 2014; Zhang et al., 2019). Nonetheless, as far as we know, scarce data are available about its antimicrobial activity against MDR A. baumannii. Gallic acid is a phenolic acid with reported antibacterial activity, especially in combination with conventional antibiotics (Shao et al., 2015; Dos Santos et al., 2018; Farrag et al., 2019). Notably, the mean minimal effective dose of cinnamic acid $(1.2 \mathrm{mg} / \mathrm{ml})$ was significantly lower than that of gallic acid $(1.67 \mathrm{mg} / \mathrm{ml})$. This significant reduction in the antimicrobial activity of gallic acid has been attributed to increased number of hydroxyl group substitutions on the benzene ring compared to cinnamic acid (Sánchez-Maldonado et al., 2011; Zhang et al., 2019). One interesting observation from the present study was the efficiency of cinnamic and gallic acids in limiting the formation of biofilm associated with MDR A. baumannii. Former studies demonstrated that gallic acid inhibited biofilm formation in Staphylococcus aureus (Luís et al., 2014), P. aeruginosa (Zhang et al., 2020), E. coli, and Streptococcus mutans (Shao et al., 2015). Likewise, the antibiofilm effect of cinnamic acid derivatives was recognized in recent studies (Zhou et al., 2020). Various mechanisms have been proposed to justify the antibiofilm activities of phenolic compounds including gallic and cinnamic acid derivatives. They may exert their effects through cleavage of peptidoglycan present in the cell wall (Burcu Bali et al., 2019) and/or inhibition of $\mathrm{N}$-acyl homo-serine lactones (AHLs)-mediated quorum sensing (Rajkumari et al., 2018; Zhang et al., 2020), coupled with the antioxidant power of these compounds, which prevents 
the formation of reactive oxygen species (ROS), and accordingly might obstruct the expression of key genes implicated in the regulation of biofilm formation (Ong et al., 2018). The SEM images reinforced the antibiofilm effect of gallic and cinnamic acids on the tested isolates. In their review about the most extensively used methods for biofilm visualization, Relucenti et al. (2021) considered SEM as the most efficient tool for comparative biofilm investigations, particularly when testing antibiofilm agents (Relucenti et al., 2021). Raorane et al. (2019) used SEM to investigate the antibiofilm effect of curcumin on Candida albicans and A. baumannii mixed biofilms and reported that SEM images of untreated biofilms showed large hyphae encasing $A$. baumannii cells; however, after treatment with $20 \mu \mathrm{g} / \mathrm{ml}$ curcumin, disintegration of biofilms was obvious, and A. baumannii cells could be clearly recognized (Raorane et al., 2019). Similarly, analysis of the obtained SEM images revealed that the treated $A$. baumannii biofilms showed distinct morphological changes compared to the untreated one. A dense matrix of intact cells was observed in the untreated biofilm, while treated ones displayed structural alterations to the matrix with visible reduction in the adherent cells along with damage to the cells. Remarkably, the damage in the structural integrity of the biofilms was more obvious in gallic-acid-treated biofilms compared to cinnamic-acid-treated ones.

\section{CONCLUSION}

This work shed the light on the debatable multifactorial correlation between biofilm formation and resistance of A. baumannii clinical isolates. The results underlined a significant association between MDR and the biofilm-forming ability. Altogether, the presence of bla $a_{\mathrm{VIM}}$ and $b l a_{\mathrm{OXA}-23}$, along with the biofilm-related genes ompA, bap, and $c s u \mathrm{E}$ influence the intensity of the formed biofilms. Exploring the relationship between antibiotic resistance and biofilm formation may unravel the complex dilemma of managing MDR pathogens, in general, and A. baumannii, in particular. Furthermore, this study

\section{REFERENCES}

Abdel-Baky, R. M., Ali, M. A., Abuo-Rahma, G. E.-D. A. A., and AbdelAziz, N. (2017). "Inhibition of urease enzyme production and some other virulence factors expression in Proteus mirabilis by $\mathrm{N}$-acetyl cysteine and dipropyl disulphide," in ed. G. Donelli Advances in Microbiology, Infectious Diseases and Public Health, (Berlin: Springer), 99-113. doi: 10.1007/5584_2016_197

Abdulzahra, A. T., Khalil, M. A., and Elkhatib, W. F. (2018). First report of colistin resistance among carbapenem-resistant Acinetobacter baumannii isolates recovered from hospitalized patients in Egypt. New Microbes New Infect. 26, 53-58. doi: 10.1016/j.nmni.2018.08.007

Abe, K., Nomura, N., and Suzuki, S. (2020). Biofilms: hot spots of horizontal gene transfer (HGT) in aquatic environments, with a focus on a new HGT mechanism. FEMS Microbiol. Ecol. 96:fiaa031. doi: 10.1093/femsec/fiaa031

Adamus-Bialek, W., Zajac, E., Parniewski, P., and Kaca, W. (2013). Comparison of antibiotic resistance patterns in collections of Escherichia coli and Proteus mirabilis uropathogenic strains. Mol. Biol. Rep. 40, 3429-3435. doi: 10.1007/ s11033-012-2420-3

Al-Shamiri, M. M., Zhang, S., Mi, P., Liu, Y., Xun, M., Yang, E., et al. (2021). Phenotypic and genotypic characteristics of Acinetobacter baumannii provided a proof that cinnamic and gallic acids exhibited inhibitory and substantial antibiofilm activities against MDR A. baumannii isolates.

\section{DATA AVAILABILITY STATEMENT}

The original contributions presented in the study are included in the article/Supplementary Material, further inquiries can be directed to the corresponding author.

\section{AUTHOR CONTRIBUTIONS}

WE, NA, and NE conceived the objectives and designed the study protocol. NA and MS provided the isolates. MS and WK participated in methodology and experimental work. NA performed the statistical analysis, prepared tables and figures, and wrote the manuscript in its required format. MS, WK, and NA analyzed the data and interpreted the results. NA, NE, and MS drafted the manuscript. WE, NA, NE, and WK revised the manuscript prior to its submission. WE chiefly supervised the study and critically revised and edited the manuscript. All authors read and approved the final submitted version.

\section{ACKNOWLEDGMENTS}

The authors hereby acknowledge the Department of Microbiology and Immunology, Faculty of Pharmacy, Ahram Canadian University (ACU), for providing us with the facilities and support required to perform the practical work.

\section{SUPPLEMENTARY MATERIAL}

The Supplementary Material for this article can be found online at: https://www.frontiersin.org/articles/10.3389/fmicb. 2021.716627/full\#supplementary-material

enrolled in the relationship among antibiotic resistance, biofilm formation and motility. Microb. Pathog. 155:104922. doi: 10.1016/j.micpath.2021. 104922

Alves, M. J., Ferreira, I. C., Froufe, H. J., Abreu, R., Martins, A., and Pintado, M. (2013). Antimicrobial activity of phenolic compounds identified in wild mushrooms, SAR analysis and docking studies. J. Appl. Microbiol. 115, 346-357. doi: $10.1111 /$ jam. 12196

Aly, M., Alsoud, N. A., Elrobh, M., Al Johani, S., and Balkhy, H. (2016). High prevalence of the PER-1 gene among carbapenem-resistant Acinetobacter baumannii in Riyadh, Saudi Arabia. Eur. J. Clin. Microbiol. Infect. Dis. 35, 1759-1766. doi: 10.1007/s10096-016-2723-8

Araújo Lima, A. V., da Silva, S. M., do Nascimento Júnior, J. A. A., Correia, M. D. S., Luz, A. C., Leal-Balbino, T. C., et al. (2020). Occurrence and diversity of intraand interhospital drug-resistant and biofilm-forming Acinetobacter baumannii and Pseudomonas aeruginosa. Microb. Drug Resist. 26, 802-814. doi: 10.1089/ mdr.2019.0214

Asaad, A. M., Ansari, S., Ajlan, S. E., and Awad, S. M. (2021). Epidemiology of biofilm producing Acinetobacter baumannii nosocomial isolates from a tertiary care hospital in Egypt: a cross-sectional study. Infect. Drug Resist. 14:709. doi: 10.2147/IDR.S261939 
Asadollahi, P., Akbari, M., Soroush, S., Taherikalani, M., Asadollahi, K., Sayehmiri, K., et al. (2012). Antimicrobial resistance patterns and their encoding genes among Acinetobacter baumannii strains isolated from burned patients. Burns 38, 1198-1203. doi: 10.1016/j.burns.2012.04.008

Azizi, O., Shakibaie, M. R., Modarresi, F., and Shahcheraghi, F. (2015). Molecular detection of class-D OXA carbapenemase genes in biofilm and non-biofilm forming clinical isolates of Acinetobacter baumannii. Jundishapur J. Microbiol. 8:e21042. doi: 10.5812/jjm.21042

Babapour, E., Haddadi, A., Mirnejad, R., Angaji, S.-A., and Amirmozafari, N. (2016). Biofilm formation in clinical isolates of nosocomial Acinetobacter baumannii and its relationship with multidrug resistance. Asian Pac. J. Trop. Biomed. 6, 528-533. doi: 10.1016/j.apjtb.2016.04.006

Badave, G. K., and Kulkarni, D. (2015). Biofilm producing multidrug resistant Acinetobacter baumannii: an emerging challenge. J. Clin. Diagn. Res. 9, DC08DC10. doi: 10.7860/JCDR/2015/11014.5398

Bagheri Josheghani, S., Moniri, R., Firoozeh, F., Sehat, M., and Dasteh Goli, Y. (2015). Susceptibility pattern and distribution of oxacillinases and blaPER1 genes among multidrug resistant Acinetobacter baumannii in a teaching hospital in Iran. J. Pathog. 2015:957259. doi: 10.1155/2015/957259

Bardbari, A. M., Arabestani, M. R., Karami, M., Keramat, F., Aghazadeh, H., Alikhani, M. Y., et al. (2018). Highly synergistic activity of melittin with imipenem and colistin in biofilm inhibition against multidrug-resistant strong biofilm producer strains of Acinetobacter baumannii. Eur. J. Clin. Microbiol. Infect. Dis. 37, 443-454. doi: 10.1007/s10096-018-3189-7

Bardbari, A. M., Arabestani, M. R., Karami, M., Keramat, F., Alikhani, M. Y., and Bagheri, K. P. (2017). Correlation between ability of biofilm formation with their responsible genes and MDR patterns in clinical and environmental Acinetobacter baumannii isolates. Microb. Pathog. 108, 122-128. doi: 10.1016/j. micpath.2017.04.039

Bocanegra-Ibarias, P., Pena-López, C., Camacho-Ortiz, A., Llaca-Díaz, J., SilvaSánchez, J., Barrios, H., et al. (2015). Genetic characterisation of drug resistance and clonal dynamics of Acinetobacter baumannii in a hospital setting in Mexico. Int. J. Antimicrob. Agents 45, 309-313. doi: 10.1016/j.ijantimicag.2014.10.022

Bradley, J.-C., Abraham, M. H., Acree, W. E., Lang, A. S. I. D., Beck, S. N., Bulger, D. A., et al. (2015). Determination of Abraham model solute descriptors for the monomeric and dimeric forms of trans-cinnamic acid using measured solubilities from the Open Notebook Science Challenge. Chem. Cent. J. 9:11. doi: 10.1186/s13065-015-0080-9

Burcu Bali, E., Erkan Türkmen, K., Erdönmez, D., and Sağlam, N. (2019). Comparative study of inhibitory potential of dietary phytochemicals against quorum sensing activity of and biofilm formation by Chromobacterium violaceum 12472, and swimming and swarming behaviour of Pseudomonas aeruginosa PAO1. Food Technol. Biotechnol. 57, 212-221. doi: 10.17113/ftb.57. 02.19 .5823

CDC (2019). Antibiotic Resistance Threats in the United States, 2019. Atlanta, GA: Department of Health and Human Services, CDC.

Chen, L., Li, H., Wen, H., Zhao, B., Niu, Y., Mo, Q., et al. (2020). Biofilm formation in Acinetobacter baumannii was inhibited by PA $\beta \mathrm{N}$ while it had no association with antibiotic resistance. Microbiologyopen 9:e1063. doi: 10.1002/mbo3. 1063

CLSI (2018). Methods for Dilution Antimicrobial Susceptibility Tests for Bacteria That Grow Aerobically (M07-Ed11), 11th Edn. Wayne, PA: Clinical and Laboratory Standards Institute.

CLSI (2019). Performance Standards for Antimicrobial Susceptibility Testing; Twenty-Ninth Informational Supplement (M100-S29). Wayne, PA: Clinical and Laboratory Standards Institute.

Coyne, S., Rosenfeld, N., Lambert, T., Courvalin, P., and Périchon, B. (2010). Overexpression of resistance-nodulation-cell division pump AdeFGH confers multidrug resistance in Acinetobacter baumannii. Antimicrob. Agents Chemother. 54, 4389-4393. doi: 10.1128/AAC.00155-10

Daneshfar, A., Ghaziaskar, H. S., and Homayoun, N. (2008). Solubility of gallic acid in methanol, ethanol, water, and ethyl acetate. J. Chem. Eng. Data 53, 776-778.

Donlan, R. M., and Costerton, J. W. (2002). Biofilms: survival mechanisms of clinically relevant microorganisms. Clin. Microbiol. Rev. 15, 167-193. doi: 10. 1128/cmr.15.2.167-193.2002

Dos Santos, J. F., Tintino, S. R., de Freitas, T. S., Campina, F. F., Irwin, R. D. A., Siqueira-Júnior, J. P., et al. (2018). In vitro e in silico evaluation of the inhibition of Staphylococcus aureus efflux pumps by caffeic and gallic acid.
Comp. Immunol. Microbiol. Infect. Dis. 57, 22-28. doi: 10.1016/j.cimid.2018. 03.001

El Far, M. Y., El-Mahallawy, H. A., and Attia, A. S. (2019). Tracing the dissemination of the international clones of multidrug-resistant Acinetobacter baumannii among cancer patients in Egypt using the PCR-based open reading frame typing (POT) method. J. Glob. Antimicrob. Resist. 19, 210-215. doi: 10.1016/j.jgar.2019.07.025

El-Din, H. T. N., Yassin, A. S., Ragab, Y. M., and Hashem, A. M. (2021). Phenotype-genotype characterization and antibiotic-resistance correlations among colonizing and infectious methicillin-resistant Staphylococcus aureus recovered from intensive care units. Infect. Drug Resist. 14:1557. doi: 10.2147/ IDR.S296000

Elham, B., and Fawzia, A. (2019). Colistin resistance in Acinetobacter baumannii isolated from critically ill patients: clinical characteristics, antimicrobial susceptibility and outcome. Afr. Health Sci. 19, 2400-2406. doi: 10.4314/ahs. v19i3.13

Elkhatib, W. F., Khalil, M. A., and Ashour, H. M. (2019). Integrons and antiseptic resistance genes mediate resistance of Acinetobacter baumannii and Pseudomonas aeruginosa isolates from intensive care unit patients with wound infections. Curr. Mol. Med. 19, 286-293.

Ellington, M. J., Kistler, J., Livermore, D. M., and Woodford, N. (2007). Multiplex PCR for rapid detection of genes encoding acquired metallo-beta-lactamases. J. Antimicrob. Chemother. 59, 321-322. doi: 10.1093/jac/dkl481

Elshikh, M., Ahmed, S., Funston, S., Dunlop, P., McGaw, M., Marchant, R., et al. (2016). Resazurin-based 96-well plate microdilution method for the determination of minimum inhibitory concentration of biosurfactants. Biotechnol. Lett. 38, 1015-1019. doi: 10.1007/s10529-0162079-2

Eze, E. C., Chenia, H. Y., and El Zowalaty, M. E. (2018). Acinetobacter baumannii biofilms: effects of physicochemical factors, virulence, antibiotic resistance determinants, gene regulation, and future antimicrobial treatments. Infect. Drug Resist. 11:2277. doi: 10.2147/IDR.S169894

Farhadi, F., Khameneh, B., Iranshahi, M., and Iranshahy, M. (2019). Antibacterial activity of flavonoids and their structure-activity relationship: an update review. Phytother. Res. 33, 13-40. doi: 10.1002/ptr.6208

Farrag, H. A., Abdallah, N., Shehata, M. M., and Awad, E. M. (2019). Natural outer membrane permeabilizers boost antibiotic action against irradiated resistant bacteria. J. Biomed. Sci. 26:69. doi: 10.1186/s12929-019-0561-6

Gottesman, T., Fedorowsky, R., Yerushalmi, R., Lellouche, J., and Nutman, A. (2021). An outbreak of carbapenem-resistant Acinetobacter baumannii in a COVID-19 dedicated hospital. Infect. Prev. Pract. 3:100113. doi: 10.1016/j. infpip.2021.100113

Guzman, J. D. (2014). Natural cinnamic acids, synthetic derivatives and hybrids with antimicrobial activity. Molecules 19, 19292-19349. doi: 10.3390/ molecules191219292

Hall, C. W., and Mah, T.-F. (2017). Molecular mechanisms of biofilm-based antibiotic resistance and tolerance in pathogenic bacteria. FEMS Microbiol. Rev. 41, 276-301. doi: 10.1093/femsre/fux010

He, X., Lu, F., Yuan, F., Jiang, D., Zhao, P., Zhu, J., et al. (2015). Biofilm formation caused by clinical Acinetobacter baumannii isolates is associated with overexpression of the AdeFGH efflux pump. Antimicrob. Agents Chemother. 59, 4817-4825. doi: 10.1128/AAC.00877-15

Heydari, S., and Eftekhar, F. (2015). Biofilm formation and $\beta$-lactamase production in burn isolates of Pseudomonas aeruginosa. Jundishapur J. Microbiol. 8:e15514. doi: 10.5812/jjm.15514

Huang, F., Fitchett, N., Razo-Gutierrez, C., Le, C., Martinez, J., Ra, G., et al. (2020). The H-NS regulator plays a role in the stress induced by carbapenemase expression in Acinetobacter baumannii. Msphere 5, e00793-20.

Kang, J., Liu, L., Liu, M., Wu, X., and Li, J. (2018). Antibacterial activity of gallic acid against Shigella flexneri and its effect on biofilm formation by repressing $\mathrm{mdoH}$ gene expression. Food Control 94, 147-154. doi: 10.1016/j.foodcont.2018. 07.011

Lee, C.-R., Lee, J. H., Park, M., Park, K. S., Bae, I. K., Kim, Y. B., et al. (2017). Biology of Acinetobacter baumannii: pathogenesis, antibiotic resistance mechanisms, and prospective treatment options. Front. Cell. Infect. Microbiol. 7:55. doi: 10. 3389/fcimb.2017.00055

Lee, H. W., Koh, Y. M., Kim, J., Lee, J. C., Lee, Y. C., Seol, S. Y., et al. (2008). Capacity of multidrug-resistant clinical isolates of Acinetobacter baumannii to 
form biofilm and adhere to epithelial cell surfaces. Clin. Microbiol. Infect. 14, 49-54. doi: 10.1111/j.1469-0691.2007.01842.x

Lemos, A. S., Campos, L. M., Melo, L., Guedes, M. C., Oliveira, L. G., Silva, T. P., et al. (2018). Antibacterial and antibiofilm activities of psychorubrin, a pyranonaphthoquinone isolated from Mitracarpus frigidus (Rubiaceae). Front. Microbiol. 9:724. doi: 10.3389/fmicb.2018.00724

Lewis, K. (2001). Riddle of biofilm resistance. Antimicrob. Agents Chemother. 45, 999-1007. doi: 10.1128/AAC.45.4.999-1007.2001

Li, Z., Ding, Z., Liu, Y., Jin, X., Xie, J., Li, T., et al. (2021). Phenotypic and genotypic characteristics of biofilm formation in clinical isolates of Acinetobacter baumannii. Infect. Drug Resist. 14:2613.

Linciano, P., Gianquinto, E., Montanari, M., Maso, L., Bellio, P., Cebrián-Sastre, E., et al. (2020). 4-Amino-1, 2, 4-triazole-3-thione as a promising scaffold for the inhibition of serine and metallo- $\beta$-lactamases. Pharmaceuticals 13:52. doi: 10.3390/ph13030052

Luís, Â, Silva, F., Sousa, S., Duarte, A. P., and Domingues, F. (2014). Antistaphylococcal and biofilm inhibitory activities of gallic, caffeic, and chlorogenic acids. Biofouling 30, 69-79. doi: 10.1080/08927014.2013.845878

Meini, M.-R., Llarrull, L. I., and Vila, A. J. (2014). Evolution of metallo$\beta$-lactamases: trends revealed by natural diversity and in vitro evolution. Antibiotics 3, 285-316. doi: 10.3390/antibiotics3030285

Mouton, J. W., Muller, A. E., Canton, R., Giske, C. G., Kahlmeter, G., and Turnidge, J. (2018). MIC-based dose adjustment: facts and fables. J. Antimicrob. Chemother. 73, 564-568.

Nahar, A., Anwar, S., and Miah, M. R. A. (2013). Association of biofilm formation with antimicrobial resistance among the Acinetobacter species in a tertiary care hospital in Bangladesh. J. Med. 14, 28-32. doi: 10.3329/jom.v14i1.14533

Ong, K. S., Mawang, C. I., Daniel-Jambun, D., Lim, Y. Y., and Lee, S. M. (2018). Current anti-biofilm strategies and potential of antioxidants in biofilm control. Expert Rev. Anti Infect. Ther. 16, 855-864. doi: 10.1080/14787210.2018.1535898

Poirel, L., Walsh, T. R., Cuvillier, V., and Nordmann, P. (2011). Multiplex PCR for detection of acquired carbapenemase genes. Diagn. Microbiol. Infect. Dis. 70, 119-123. doi: 10.1016/j.diagmicrobio.2010.12.002

Qi, L., Li, H., Zhang, C., Liang, B., Li, J., Wang, L., et al. (2016). Relationship between antibiotic resistance, biofilm formation, and biofilm-specific resistance in Acinetobacter baumannii. Front. Microbiol. 7:483. doi: 10.3389/fmicb.2016. 00483

Rajkumari, J., Borkotoky, S., Murali, A., Suchiang, K., Mohanty, S. K., and Busi, S. (2018). Cinnamic acid attenuates quorum sensing associated virulence factors and biofilm formation in Pseudomonas aeruginosa PAO1. Biotechnol. Lett. 40, 1087-1100. doi: 10.1007/s10529-018-2557-9

Ranjbar, R., and Farahani, A. (2019). Study of genetic diversity, biofilm formation, and detection of Carbapenemase, MBL, ESBL, and tetracycline resistance genes in multidrug-resistant Acinetobacter baumannii isolated from burn wound infections in Iran. Antimicrob. Resist. Infect. Control. 8:172. doi: 10.1186/ s13756-019-0612-5

Raorane, C. J., Lee, J.-H., Kim, Y.-G., Rajasekharan, S. K., García-Contreras, R., and Lee, J. (2019). Antibiofilm and antivirulence efficacies of flavonoids and curcumin against Acinetobacter baumannii. Front. Microbiol. 10:990. doi: 10. 3389/fmicb.2019.00990

Relucenti, M., Familiari, G., Donfrancesco, O., Taurino, M., Li, X., Chen, R., et al. (2021). Microscopy methods for biofilm imaging: focus on SEM and VP-SEM pros and cons. Biology 10:51.

Sánchez-Maldonado, A., Schieber, A., and Gänzle, M. (2011). Structure-function relationships of the antibacterial activity of phenolic acids and their metabolism by lactic acid bacteria. J. Appl. Microbiol. 111, 1176-1184. doi: 10.1111/j.13652672.2011.05141.x

Shao, D., Li, J., Li, J., Tang, R., Liu, L., Shi, J., et al. (2015). Inhibition of gallic acid on the growth and biofilm formation of Escherichia coli and Streptococcus mutans. J. Food Sci. 80, M1299-M1305. doi: 10.1111/1750-3841.12902

Shenkutie, A. M., Yao, M. Z., Siu, G.K.-h, Wong, B. K. C., and Leung, P.H.$\mathrm{m}$ (2020). Biofilm-induced antibiotic resistance in clinical Acinetobacter baumannii isolates. Antibiotics 9:817. doi: 10.3390/antibiotics9110817

Sung, J. Y. (2018). Molecular characterization and antimicrobial susceptibility of biofilm-forming Acinetobacter baumannii clinical isolates from Daejeon, Korea. Korean J. Clin. Lab. Sci. 50, 100-109. doi: 10.15324/kjcls.2018.50.2.100

Tchuinte, P. L. S., Rabenandrasana, M. A. N., Kowalewicz, C., Andrianoelina, V. H., Rakotondrasoa, A., Andrianirina, Z. Z., et al. (2019). Phenotypic and molecular characterisations of carbapenem-resistant Acinetobacter baumannii strains isolated in Madagascar. Antimicrob. Resist. Infect. Control 8:31. doi: 10.1186/s13756-019-0491-9

Thet, K. T., Lunha, K., Srisrattakarn, A., Lulitanond, A., Tavichakorntrakool, R., Kuwatjanakul, W., et al. (2020). Colistin heteroresistance in carbapenemresistant Acinetobacter baumannii clinical isolates from a Thai university hospital. World J. Microbiol. Biotechnol. 36:102. doi: 10.1007/s11274-02002873-8

Tutar, U., Çelik, C., Karaman, Ý, Atas, M., and Hepokur, C. (2016). Anti-biofilm and antimicrobial activity of Mentha pulegium L essential oil against multidrugresistant Acinetobacter baumannii. Trop. J. Pharm. Res. 15:1039. doi: 10.4314/ tjpr.v15i5.20

Uruén, C., Chopo-Escuin, G., Tommassen, J., Mainar-Jaime, R. C., and Arenas, J. (2021). Biofilms as promoters of bacterial antibiotic resistance and tolerance. Antibiotics 10:3. doi: 10.3390/antibiotics10010003

Vestby, L. K., Grønseth, T., Simm, R., and Nesse, L. L. (2020). Bacterial biofilm and its role in the pathogenesis of disease. Antibiotics 9:59. doi: 10.3390/ antibiotics 9020059

WHO (2017). Prioritization of Pathogens to Guide Discovery, Research and Development of New Antibiotics for Drug Resistant Bacterial Infections, Including Tuberculosis 2017 November 1, 2019. Available online at: https://www.who.int/ medicines/areas/rational_use/prioritization-of-pathogens/en/ (accessed Nov 1, 2019)

Xie, R., Shao, N., and Zheng, J. (2020). Integrated co-functional network analysis on the resistance and virulence features in Acinetobacter baumannii. Front. Microbiol. 11:2677. doi: 10.3389/fmicb.2020.598380

Yang, C.-H., Su, P.-W., Moi, S.-H., and Chuang, L.-Y. (2019). Biofilm formation in Acinetobacter baumannii: genotype-phenotype correlation. Molecules 24:1849. doi: 10.3390/molecules 24101849

Yu, Y.-s, Zhou, H., Yang, Q., Chen, Y.-g, and Li, L.-j (2007). Widespread occurrence of aminoglycoside resistance due to ArmA methylase in imipenem-resistant Acinetobacter baumannii isolates in China. J. Antimicrob. Chemother. 60, 454455. doi: $10.1093 / \mathrm{jac} / \mathrm{dkm} 208$

Zeighami, H., Valadkhani, F., Shapouri, R., Samadi, E., and Haghi, F. (2019). Virulence characteristics of multidrug resistant biofilm forming Acinetobacter baumannii isolated from intensive care unit patients. BMC Infect. Dis. 19:629. doi: 10.1186/s12879-019-4272-0

Zhang, J., Xu, F., Yao, L., Wang, L., Wang, M., and Wang, G. (2020). Ethanol extract of Campsis grandiflora flower and its organic acid components have inhibitory effects on autoinducer type 1 quorum sensing. Molecules 25:4727. doi: 10.3390/molecules 25204727

Zhang, Y., Wei, J., Qiu, Y., Niu, C., Song, Z., Yuan, Y., et al. (2019). Structuredependent inhibition of Stenotrophomonas maltophilia by polyphenol and its impact on cell membrane. Front. Microbiol. 10:2646. doi: 10.3389/fmicb.2019. 02646

Zhou, L., Zhang, Y., Ge, Y., Zhu, X., and Pan, J. (2020). Regulatory mechanisms and promising applications of quorum sensing-inhibiting agents in control of bacterial biofilm formation. Front. Microbiol. 11:2558. doi: 10.3389/fmicb.2020. 589640

Zhu, X.-Q., Liu, Y.-Y., Wu, R., Xun, H., Sun, J., Li, J., et al. (2021). Impact of mcr-1 on the development of high level colistin resistance in Klebsiella pneumoniae and Escherichia coli. Front. Microbiol. 12:878.

Conflict of Interest: The authors declare that the research was conducted in the absence of any commercial or financial relationships that could be construed as a potential conflict of interest.

Publisher's Note: All claims expressed in this article are solely those of the authors and do not necessarily represent those of their affiliated organizations, or those of the publisher, the editors and the reviewers. Any product that may be evaluated in this article, or claim that may be made by its manufacturer, is not guaranteed or endorsed by the publisher.

Copyright (c) 2021 Sherif, Elkhatib, Khalaf, Elleboudy and Abdelaziz. This is an open-access article distributed under the terms of the Creative Commons Attribution License (CC BY). The use, distribution or reproduction in other forums is permitted, provided the original author(s) and the copyright owner(s) are credited and that the original publication in this journal is cited, in accordance with accepted academic practice. No use, distribution or reproduction is permitted which does not comply with these terms. 